\title{
Tents, Chairs, Tacos, Kites, and Rods: Shapes and Plasmonic Properties of Singly Twinned Magnesium Nanoparticles
}

Jérémie Asselin, $\triangle$ Christina Boukouvala, $\triangle$ Elizabeth R. Hopper, Quentin M. Ramasse, John S. Biggins, and Emilie Ringe*

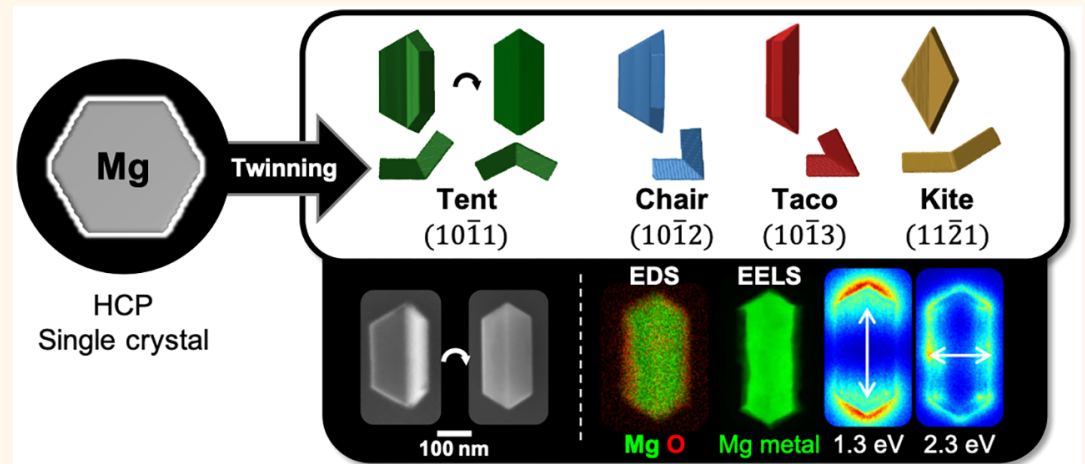

ABSTRACT: Nanostructures of some metals can sustain light-driven electron oscillations called localized surface plasmon resonances, or LSPRs, that give rise to absorption, scattering, and local electric field enhancement. Their resonant frequency is dictated by the nanoparticle (NP) shape and size, fueling much research geared toward discovery and control of new structures. LSPR properties also depend on composition; traditional, rare, and expensive noble metals ( $\mathrm{Ag}, \mathrm{Au}$ ) are increasingly eclipsed by earth-abundant alternatives, with $\mathrm{Mg}$ being an exciting candidate capable of sustaining resonances across the ultraviolet, visible, and near-infrared spectral ranges. Here, we report numerical predictions and experimental verifications of a set of shapes based on $\mathrm{Mg}$ NPs displaying various twinning patterns including $(10 \overline{1} 1),(10 \overline{1} 2),(10 \overline{1} 3)$, and $(11 \overline{2} 1)$, that create tent-, chair-, taco-, and kite-shaped NPs, respectively. These are strikingly different from what is obtained for typical plasmonic metals because $\mathrm{Mg}$ crystallizes in a hexagonal close packed structure, as opposed to the cubic $\mathrm{Al}, \mathrm{Cu}, \mathrm{Ag}$, and $\mathrm{Au}$. A numerical survey of the optical response of the various structures, as well as the effect of size and aspect ratio, reveals their rich array of resonances, which are supported by single-particle optical scattering experiments. Further, corresponding numerical and experimental studies of the near-field plasmon distribution via scanning transmission electron microscopy electron-energy loss spectroscopy unravels a mode nature and distribution that are unlike those of either hexagonal plates or cylindrical rods. These NPs, made from earth-abundant $\mathrm{Mg}$, provide interesting ways to control light at the nanoscale across the ultraviolet, visible, and near-infrared spectral ranges.

KEYWORDS: magnesium nanoparticles, nanoplasmonics, localized surface plasmon resonance, nanoparticle shape, Wulff construction, electron-energy loss spectroscopy

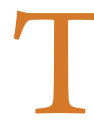
he optical response of nanomaterials has attracted much attention in the past decades owing to exquisite tuning opportunities afforded by size, shape, and composition manipulation. Nanoparticles (NPs) of some metals, traditionally $\mathrm{Ag}$ and $\mathrm{Au}$, exhibit an intrinsically nanoscale phenomenon called localized surface plasmon resonance (LSPR). This strong light-matter interaction results from the coupling of the oscillating electric field of light with
Received: February 18, 2020

Accepted: April 14, 2020

Published: April 14, 2020 
a) HCP twin planes

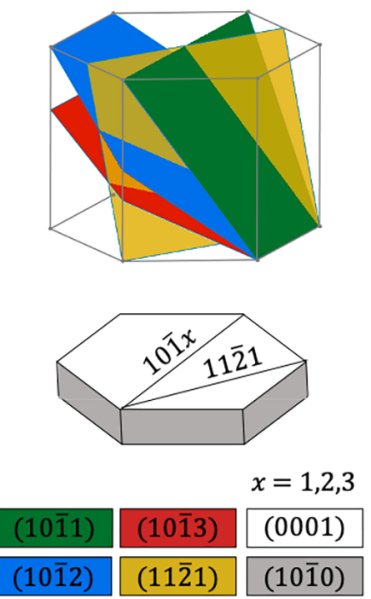

b) Single crystal Plate
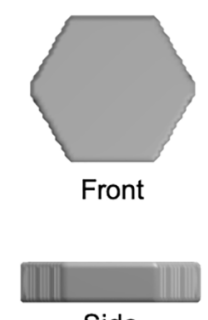

Side

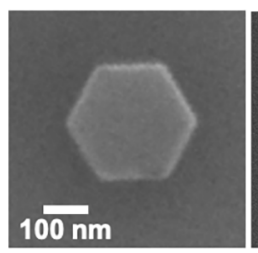

c) $10 \overline{1} 1$ twin

Tent
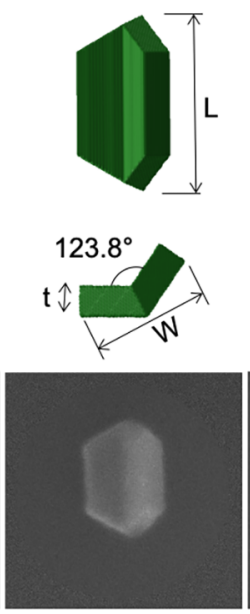

d) $10 \overline{1} 2$ twin Chair

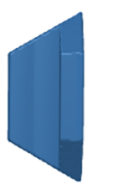

$86.4^{\circ}$

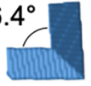

e) $10 \overline{1} 3$ twin Taco

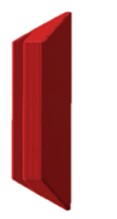

$64^{\circ} \alpha$ f) $11 \overline{2} 1$ twin

Kite

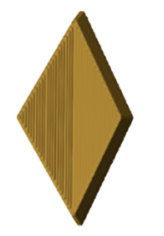

$145.8^{\circ}$
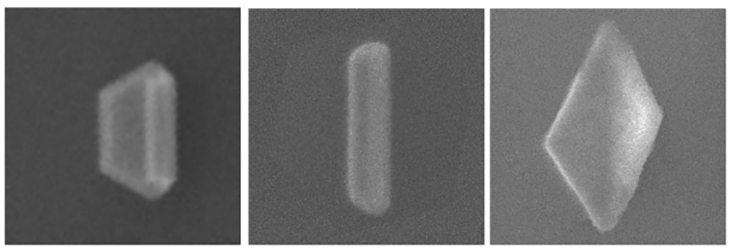

Figure 1. Shapes of Mg crystals. (a) Unit cell arrangement in HCP crystals and representation of the multiple low-energy twinning planes. (b) Predicted Wulff shapes for single-crystalline and (c-f) singly twinned structures with predominant (0001) facets; the twin planes are noted above the structure, and parameters used in the calculations are reported in Table S1. SEM images of the experimentally obtained shapes are placed below the models; additional images reported in Figures S2-S6.

the cloud of delocalized conduction electrons inside an NP smaller than the wavelength of light. LSPRs are of interest because they localize electric fields, enabling surface-enhanced spectroscopies and local refractive index sensing, for instance. $^{1-3}$ Recent results demonstrate that this resonance phenomenon can also be utilized as an antenna to capture light and subsequently transfer its energy in the form of "hot" (nonthermally equilibrated) charge carriers or heat, to drive chemical and physical processes. ${ }^{4-8}$

The LSPR frequency and bandwidth tuning required to optimize an NP's performance for a given application is achieved mainly through choice of shape, size, and composition. $^{9-11}$ Shape is a powerful tool, as it further allows control of the locally enhanced electric field through the creation of multiple resonance modes that have different energies and enhancement distributions. For instance, a cylindrical rod can sustain multiple longitudinal modes with an increasing number of nodes (like modes of a string) as well as a transverse mode. ${ }^{12-15}$ Another example is the field distribution around a cube, where corners, edges, and faces experience enhancement at different energies. ${ }^{16}$

Because shape contributes so much to the properties of plasmonic materials, it has been and remains a central theme of nanoscience research. The final shape of an NP is dictated by its nuclei symmetry and the facets predominantly expressed. The former includes the twinning pattern of the NP, dictated by the twin energies specific to the crystal structure. Within each twinning pattern, the latter, i.e., the relative surface energy or growth velocity of different crystallographic facets, then determines the final shape according to the Wulff construction. $^{17-20}$

Well-known examples of the use of twinning and Wulff construction are found for NPs of face-centered cubic (FCC) materials $(\mathrm{Ag}, \mathrm{Au}, \mathrm{Cu}, \mathrm{Al}) .^{21,22}$ These have low (111) twin energy and can exist as singly twinned (bipyramids), ${ }^{23} 5$-fold twinned (pentagonal structures), ${ }^{24,25}$ or 20 -fold twins (icosahedra). ${ }^{24}$ Further, the close-packed FCC (111) facets are the most thermodynamically stable followed by the (100), leading to shapes close to cuboctahedra and pentagonal bipyramids for single crystals and 5-fold twins, respectively. Relative surface energies can be manipulated by the growth environment, by, for example, adding surfactants ${ }^{26-28}$ or coordinating atoms ${ }^{29,30}$ to achieve a plethora of shapes including cubes, stars, and hexagonal and triangular platelets, to name a few. Kinetic effects during growth of twinned structures, which favor atomic deposition in concave and/or defective sites, further control the final NP shape, explaining for instance why sharp decahedra are overwhelmingly observed instead of the re-entrant surfaces expected for the thermodynamically predicted Marks decahedra. ${ }^{19,31,32}$

A systematic understanding of NP shape for structures other than the dominant FCC materials is scarce in the nanotechnology community. ${ }^{33,34}$ Meanwhile, there is increased research and interest in alternative metals for nanoscale phenomena including plasmonics and catalysis, and not all the newcomers crystallize in FCC. Hexagonal close packed (HCP) $\mathrm{Mg}$, for instance, has attracted much interest as a plasmonic metal because of its low losses across the ultraviolet, visible, and near-infrared ${ }^{35-38}$ electromagnetic ranges and its abundance in earth's crust. Its optical properties were first described from studies of fabricated structures such as helices and nanodisks; recently the plasmonic activity of colloidally synthesized Mg NPs and decorated Mg NPs was reported. ${ }^{35,39}$ In parallel, the number of reported syntheses of metallic $\mathrm{Mg}$ NPs, focused on hydrogen or energy storage applications, has bloomed in the past decade. ${ }^{40-46}$

A commonly observed $\mathrm{Mg} \mathrm{NP}$ shape is a single-crystal hexagonal plate predominantly displaying the close-packed, hence stable, (0001) facets. Yet a number of other shapes are apparent in reaction mixtures and early work on evaporationgrown submicron particles. ${ }^{44,47,48}$ This has been attributed, in some works, to twinning, which can occur along multiple planes in HCP crystals, ${ }^{49,50}$ leading to a potentially large number of low-energy shapes.

In this work, we present a comprehensive study of different Mg NP shapes, from crystallographic prediction to experimental synthesis and optical behavior. First, the Wulff construction model shows folded shapes based on single- 
crystal and twinned HCP $\mathrm{Mg}$. We describe the relative distribution of these structures from hundreds of NPs in a colloidal mixture, obtained from the reduction of an organometallic $\mathrm{Mg}$ precursor. Second, we have characterized the exact shape of these NPs via complementary electron microscopy and tomographic approaches, leading to a comprehensive understanding of their structures showing a thorough match with the predicted shapes. The NPs were confirmed to be metallic $\mathrm{Mg}$ covered by a thin oxide layer, as previously reported. ${ }^{35,39}$ Third, a numerical survey of the optical response of the various structures, as well as the effect of size and aspect ratio, revealed their rich array of resonances, which are supported by experimental single-particle scattering results. Finally, numerical and experimental studies of the near-field plasmon distribution via electron-energy loss spectroscopy unraveled a mode nature and distribution that are unlike those of either hexagonal plates or cylindrical rods. These four main results, namely, shape prediction, shape characterization, farfield scattering properties, and near-field light localization, are presented in this order. These NPs, made from earth-abundant $\mathrm{Mg}$, provide interesting ways to control light at the nanoscale across the ultraviolet, visible, and near-infrared spectral ranges.

\section{RESULTS/DISCUSSION}

Shapes of Twinned Mg Nanoparticles. Mg, unlike the other common plasmonic metals $\mathrm{Au}, \mathrm{Ag}, \mathrm{Al}$, and $\mathrm{Cu}$, crystallizes in an HCP structure and has several low-energy twin planes, enabling shapes in striking contrast with those regularly observed in the FCC metals. Here, these shapes are studied numerically by adapting the Wulff construction to HCP, then experimentally verified via complementary characterization techniques deployed on products obtained from an air-free solution synthesis.

Shape Prediction with the Wulff Construction. The unit cell of $\mathrm{Mg}$ is hexagonal with $a=3.19 \AA$ and $c=5.18 \AA$, giving a $c / a$ ratio of 1.62 , near that of perfect $\mathrm{HCP}$ packing of hard spheres (1.63). This atomic arrangement yields closepacked (0001) facets, which are lowest in surface energy as well as densely packed $(10 \overline{1} 0)$ and $(10 \overline{1} 1)$ also of low energy, as confirmed by numerical calculations. ${ }^{51}$ Further, HCP allows twinning along the (10 $\overline{1} x), x=1,2,3$, and $(11 \overline{2} y), y=1,2,3,4$ planes, ${ }^{49}$ some of which $(x=1,2,3$ and $y=1)$ are shown in Figure 1a.

The relative surface energy and orientation of a twinning plane dictates the shape of crystals, as explained by the Wulff construction and its adaptation to twinned structures. ${ }^{18,19}$ Wulff models are well established for cubic materials because of the structure's simplicity and importance in nanotechnology; ${ }^{18,20,52,53}$ HCP structures have not received the same level of attention, and shape modeling tools encoding the crystallography and twinning of HCP are not straightforwardly available. To predict shapes achievable in $\mathrm{Mg}$, we first adapted the Wulff construction approach we published earlier ${ }^{19,20}$ to the hexagonal system by coding appropriate axis and plane directions, then added the possibility of twinning along each of the low-energy twin planes shown in Figure 1a. We then performed numerical predictions of the shape of crystals by varying the relative surface energies and kinetic growth enhancement in the presence of a twin plane. ${ }^{19}$

A simple shape for HCP is a hexagonal platelet; this occurs in the case of single crystals and has often been observed. $^{35,47,54}$ From this shape one can visualize the constituents of a twinned structure: twins effectively "cut" the single crystal shape and create a symmetry plane. ${ }^{18}$ The twins $(10 \overline{1} 1),(10 \overline{1} 2),(10 \overline{1} 3)$, and $(11 \overline{2} 1)$ are at an angle of $61.9^{\circ}, 43.2^{\circ}, 32.0^{\circ}$, and $72.9^{\circ}$, respectively, from the basal (0001) plane (Figure 1). Singly twinned particle shapes are created through joining these constituting segments at the appropriate crystallographic angle, as described by Marks for FCC structures. ${ }^{18}$

Shapes obtained by varying the twinning pattern, while considering the expected kinetic growth enhancement due to the presence of strain and defects around the twin plane, are shown in Figure 1. These are noticeably different from shapes commonly observed in other plasmonic materials. The particle obtained with the $(10 \overline{1} 1)$ twin plane has two segments with three $(10 \overline{10})$ facets and two (0001) facets (in addition to the twin plane) that come together at a $123.8^{\circ}$ angle, leading to a $\mathrm{V}$-shaped configuration one might recognize, in Europe, as a Canadian tent and elsewhere as a tent. Similarly, the geometry resulting from a (1012)-twin plane is also that of two six-facet segments with predominant (0001); these come together at an angle of $86.4^{\circ}$, resembling a straight chair similar to a foldable patio chair. A much sharper angle is obtained from the (10혀) twinned structure and shows a shrinking of the length of the $(10 \overline{1} 0)$ closest to the twin plane; this structure can be described as, keeping with the picnic theme, a taco. Lastly, the angle associated with the $(11 \overline{21})$ twin plane creates segments that have, in addition to the twin plane, two (0001) and only two (1010) facets; they join at an angle of $145.8^{\circ}$ and form a folded kite structure.

Some of these structures have been reported by Ohno and Yamauchi, ${ }^{47}$ who, in 1981, described nano- to microstructures of $\mathrm{Mg}$ created by evaporation in a noble gas environment $(\mathrm{He}$, $\mathrm{Ne}, \mathrm{Ar}$, or $\mathrm{Xe}$ ). They directly described the kite structure with its facets and angles, and several of the particles they reported but did not discuss could be interpreted in light of our calculation as tents. Further, Ohno and Yamauchi describe

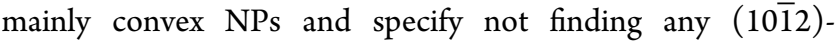
twinned structures. Further, the shapes reported here are exciting because of their anisotropy (aspect ratio, AR > 1) which results in structures unlike those of single crystals or 5fold twinned FCC crystals.

Rods (AR > 2.2) are observed in the Wulff model by enhancing growth in the concave cusp of the re-entrant edges $\left(<90^{\circ}\right)$ of the $(10 \overline{1} 2)$ twinned chair, as shown in Figure S1. This is analogous to the filled (10 $\overline{1} 1)$-twinned (filled tent) and filled (10 $\overline{1} 3$ )-twinned (filled taco, Figure S1) structures described by Ohno and Yamauchi, which can be extended in length by allowing twin kinetic enhancement. Experimentally differentiating between these structures can be difficult in a 2D projection such as a high angle annular dark field scanning transmission electron microscope (HAADF-STEM) image; however, 3D tomographic reconstruction (vide infra) confirms the existence of the filled chair. Several rods seem devoid of a twin plane, as concluded from the lack of contrast change from one segment to another in TEM (due to diffraction contrast) and lack of prominent ridge from the pseudo-3D imaging with secondary electrons (SE) in a scanning electron microscope (SEM). They appear to be truncated hexagonal plates, a shape also present (but not discussed) in the pictures reported by Ohno and Yamauchi.

Experimental Shapes. To understand the range of shapes expressed experimentally, we synthesized Mg NPs in solution, surmounting the difficulties associated with the symmetrybreaking presence of a substrate during growth. We achieved 
100-400 nm long colloidal NPs by reducing dibutyl $n$ magnesium by lithium naphthalenide in dry tetrahydrofuran. A wide range of shapes are observed; all predominantly express the close packed (0001) facets but have different shapes owing to the presence of differently oriented twin planes. Further to their different shapes, when deposited on a flat substrate, most of the NPs can adopt one of two conformations. First, the NP can lay with the folding bisector perpendicular to the substrate, as in the top NP of Figure 2a; this will be called $\Lambda$ and is the
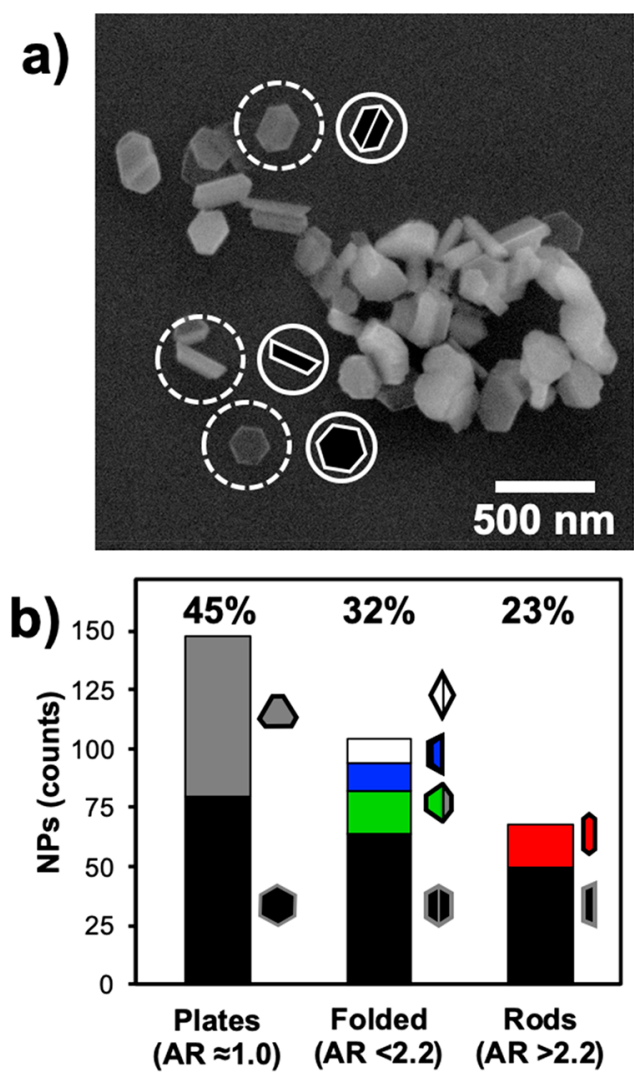

Figure 2. Distribution of NP shapes in colloidal Mg NPs. (a) Representative SEM image; additional images in Figures S3-S6. (b) Shape distribution from $N=320 \mathrm{NPs}$, grouped by aspect ratio, then by shape. Plates $(A R \approx 1)$ include hexagons and truncated hexagons; folded structures $(\mathrm{AR}<2.2)$ include kites (white), tacos (blue), chairs (green), and tents (black); rods (AR > 2.2) include filled chairs (black) and truncated plates (red).

highest symmetry arrangement. The other configuration is with one of the (0001) faces lying flat on the substrate, as is shown for all of the NPs in Figure 1; we will call this the $\geq$ configuration. These configurations can be readily distinguished by the contrast in SEM and STEM or by the intensity of the $\mathrm{Mg} \mathrm{K} \alpha$ in an energy dispersive X-ray spectroscopy (EDS) linescan or map, all linked to their different thickness profile. Indeed, $\Lambda$ is symmetrical and $\geq$ is not (Figure S2). For hexagonal plates without folds these configurations are, of course, the same.

The reaction mixture distribution, obtained by measuring 320 NPs spanning various sizes, shapes, and configurations, is shown in Figure 2b, with additional large-field SEM images in Figures S3-S5. To quantify the size of the NPs, we define aspect ratio (AR) as the length over the projected width in the $\Lambda$ configuration, as shown in Figure 1c (the AR of NPs in the $\geq$ configuration is calculated accordingly). The structures observed naturally fall into three ranges of aspect ratio: the plates $(\mathrm{AR} \approx 1)$ include thin hexagons and truncated triangles; the folded structures $(1.2<\mathrm{AR}<2.2)$ comprise the tents, chairs, tacos, and kites; the rods contain the elongated and filled structures with $A R>2.2$. The single-crystal hexagonal plates account for $25 \%$ and the truncated triangles, $20 \%$ (total for plates, $45 \%)$. About a third (32\%) of the NPs are folded structures, with $20 \%$ tents, $6 \%$ chairs, $4 \%$ tacos, and $2 \%$ kites. This population distribution matches well the relative energy of the twin planes, with the $(10 \overline{1} 1)$ twin plane being the lowest energy; hence tent NPs are most prevalent. ${ }^{55,56}$ No $(11 \overline{2} y), y>$ 1 , were unequivocally identified in the experimental mixture. Lastly, $23 \%$ of the structures were found to have an AR greater than 2.2; these are the filled chairs and truncated hexagons, with $16 \%$ and $7 \%$, respectively. However, distinguishing the two types of elongated structures based on a $2 \mathrm{D}$ projection is difficult, leading to a significant uncertainty in this distribution.

The shape of the NPs obtained experimentally match very well the predictions of the Wulff model, as supported by results from multiple complementary techniques including SEM (Figures 1, 2, and S3-S5), SEM at multiple tilt angles (Figure S6), TEM (Figure S3), STEM-HAADF (Figures 3, 4, 11, and

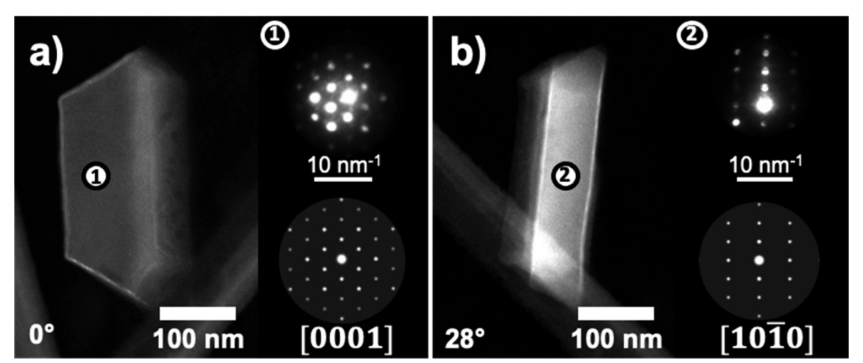

Figure 3. Shape and crystallography of a $\mathrm{Mg}$ tent $((10 \overline{1} 1)$ twin, AR $=2.0$ ), sitting in the $\geq$ configuration on the substrate. STEMHAADF and experimental (top) and simulated (bottom) electron diffraction patterns at (a) $0^{\circ}$ tilt angle and (b) $28^{\circ}$ tilt angle.

S2 and S18), and STEM-EDS linescans and maps (Figures 4 and S2). To further support this match, 3D reconstructions of the shapes of multiple NPs were obtained using STEMHAADF tomography (Figures S7 and S8). The short NP depicted in Figure 3 is folded with an angle of $123.9^{\circ}$ and an aspect ratio of 2.0 , in excellent agreement with the predicted tent shape, i.e., $(10 \overline{1} 1)$ twinning with a folding angle of $123.8^{\circ}$ (Figure S7). A large aspect ratio $\mathrm{NP}(\mathrm{AR}=4.5)$ was also reconstructed (Figure S8) and displays a convex, nearrectangular cross-section consistent with the filled chair shape (Figure S1).

To elucidate the relative orientation of the segments in folded NPs, electron diffraction patterns were obtained in STEM mode with a small convergence angle in order to probe the local crystallography. Shown in Figure 3, these reveal that the two segments of the tent NP indeed have different orientations, confirming that the NP is not a single crystal. The segment with facets parallel to the substrate shows a pattern consistent with the beam propagating along the [0001] direction; that is, the facets exposed are (0001). The diffraction from the other segment, taken with the particle tilted $28^{\circ}$ around the long axis of the NP, reveals that the perpendicular direction to the long edge side facet of the segment standing proud from the surface is $[10 \overline{1} 0]$, confirming the $(10 \overline{1} 1)$ 


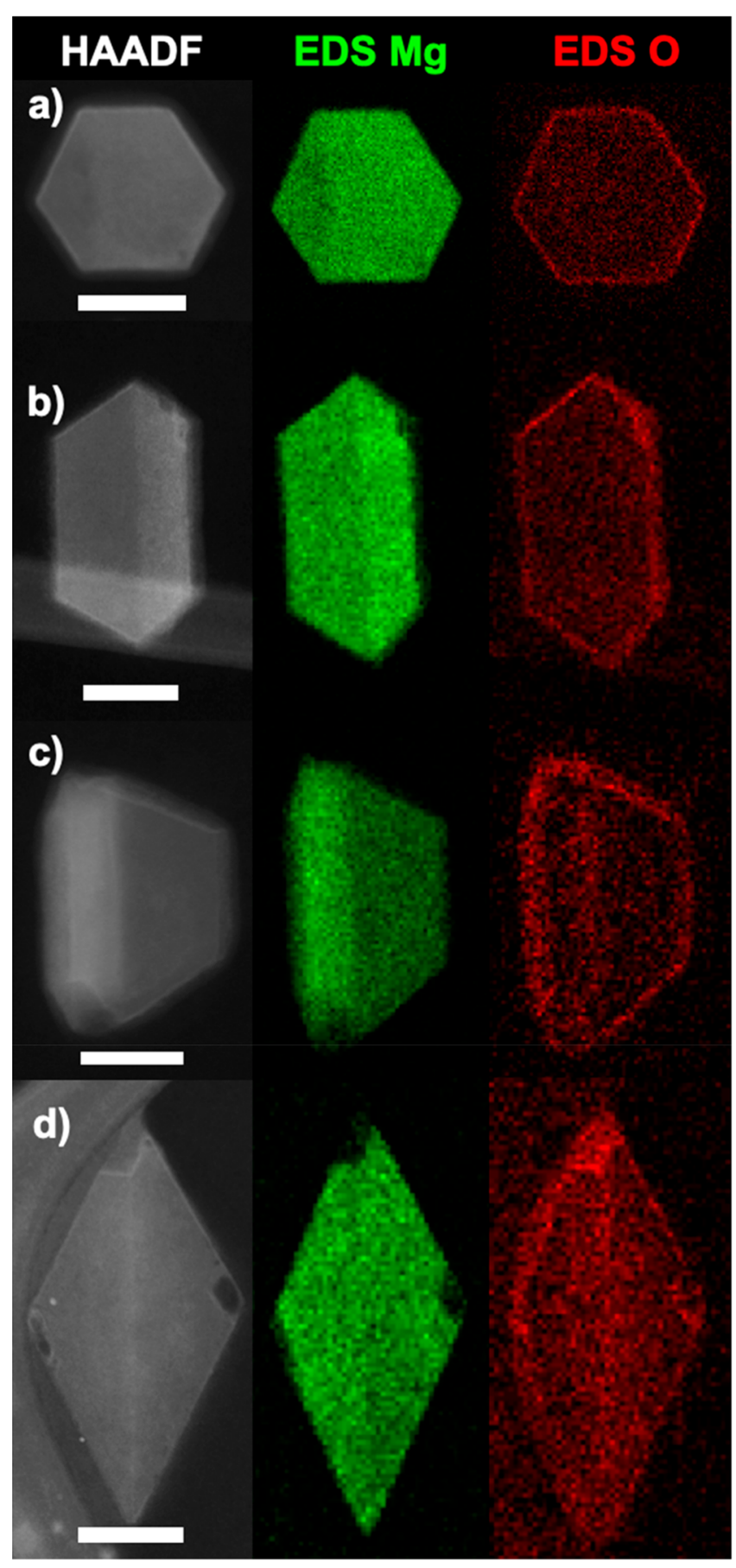

Figure 4. Elemental composition maps of Mg NPs. Intensity maps of STEM-HAADF, $\mathrm{Mg} \mathrm{K} \alpha$, and O K $\alpha$ STEM-EDS for a (a) single crystal hexagon, (b) tent, (c) chair, and (d) kite. The oxide layer appears to be 5-8 nm thick; scale bars, $100 \mathrm{~nm}$. Additional maps are reported in Figure S2.

nature of the twinning. Further results for a long rod are reported in Figure S8.

The thickness of the NPs is more difficult to assess; tomography indicates, for the NP in Figure 3, a thickness of 51 nm. Further, several STEM/SEM images of NP aggregates allow viewing in different orientations; from those we estimate a thickness, including the oxide layer, of $36 \pm 7 \mathrm{~nm}$ (measured on 26 tent NPs, Figure S9). The minimum and maximum thicknesses observed are 24 and $51 \mathrm{~nm}$, respectively.
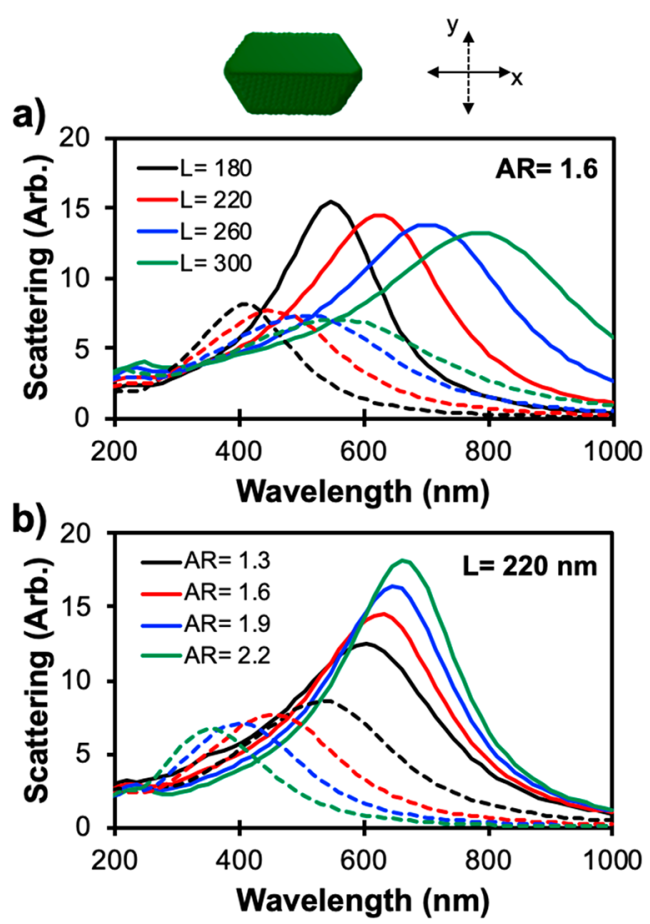

Figure 5. Effect of length $(L)$ and aspect ratio (AR) on the optical scattering of Mg tent NPs. Calculated optical scattering for (a) varying lengths with fixed $A R=1.6$ and (b) varying aspect ratios with fixed $L=220 \mathrm{~nm}$. The $x$ polarization (longitudinal) is shown in solid lines and $y$ (transverse), in dashed lines. The NPs are bare $\mathrm{Mg}$ under vacuum; the incident field is along the NP bisector $(z$ axis). Averaged spectra are reported in Figure S10.

Regardless of shape, orientation, or thickness, these nanocrystals are formed of a core of metallic, plasmonic $\mathrm{Mg}$ covered by a spontaneously formed, protective magnesium oxide layer. We confirm the presence of this thin oxide with STEM-EDS maps (Figures 4 and S2): $\mathrm{Mg}$ is present in the entire $\mathrm{NP}$, while $\mathrm{O}$ is mainly at the edges with some intensity all over the NP because of the 2D projection of STEM imaging. The oxide thickness appears to be $5-10 \mathrm{~nm}$, likely an upper bound owing to the delocalization of EDS. This is consistent with previously reported core-loss EELS maps showing an oxide thickness of $5-8 \mathrm{~nm} .^{35}$ This oxide protects the NP from full oxidation, and the core $\mathrm{Mg}$ remains metallic, as confirmed by the presence of the bulk plasmon peak, which is characteristic of metallic $\mathrm{Mg}$ at $10.6 \mathrm{eV}$. This metallic core bestows these NPs plasmonic properties.

Numerical and Experimental Studies of Optical Properties. Mg NPs of various shapes have a plethora of interesting plasmonic properties. We utilized an HCP and twinned variant of our previously developed graphical user interface to turn the Wulff shapes described above into an array of dipoles describing the NPs. ${ }^{20}$ We then use those to numerically solve Maxwell's equations for a plane wave and an electron beam excitation. Coupled with experimental results from both optical and electron beam excitations, these reveal the presence of multiple size- and shape-dependent plasmon resonances across the ultraviolet, visible, and near-infrared spectral ranges.

Numerical Results: Far-Field Optical Scattering. Optical scattering simulations, performed in the discrete dipole approximation (DDA $)^{57}$ on dipole arrays obtained directly from our twinned HCP Wulff model reveal the effect 


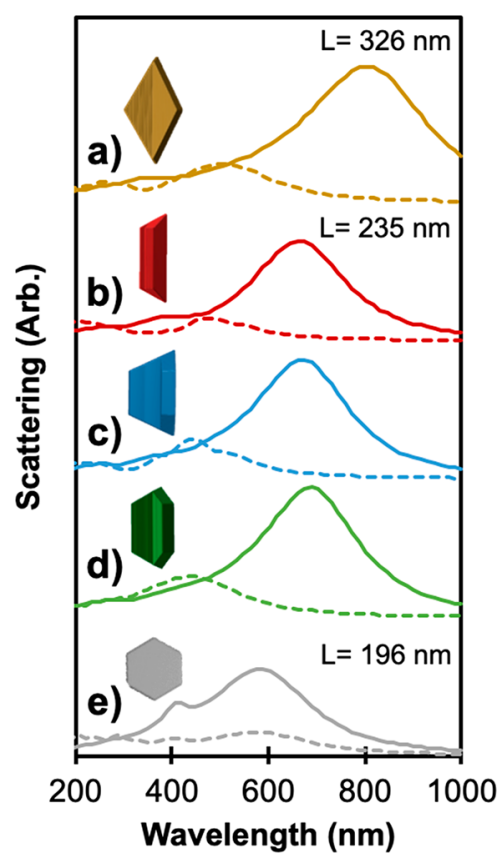

Figure 6. Effect of shape on the optical scattering of equal total volume Mg NPs coated with a $\sim 4.5 \mathrm{~nm} \mathrm{MgO}$ layer under vacuum. Calculated optical scattering intensities for different $\mathrm{Mg}$ shapes with longitudinal (solid lines) and transverse (dashed lines) polarizations, for a (a) kite, (b) taco, (c) chair, (d) tent, and (e) hexagonal plate. The length $(L)$ varies as indicated and is the same for $(\mathrm{b})-(\mathrm{d})$; the incident field is tilted $31^{\circ}$ from the substrate, as shown in Figure S13. Additional calculated spectra are reported in Figure S13.

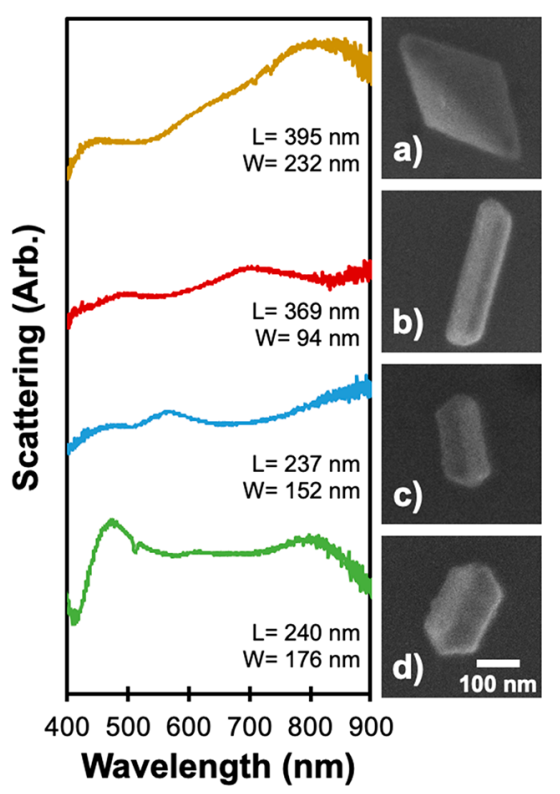

Figure 7. Experimental optical scattering intensities for Mg NPs of various shapes. From top to bottom a (a) kite, (b) taco, (c) chair, and (d) tent in the $\geq$ configuration. Length $(L)$ and width $(W)$ vary as indicated.

of size and shape on the plasmonic response of Mg NPs. Here, scattering is displayed rather than extinction or absorption as it is measured experimentally; absorption and extinction data show equivalent trends and are available upon request. The optical scattering spectra (Figures 5 and S10) show a low-

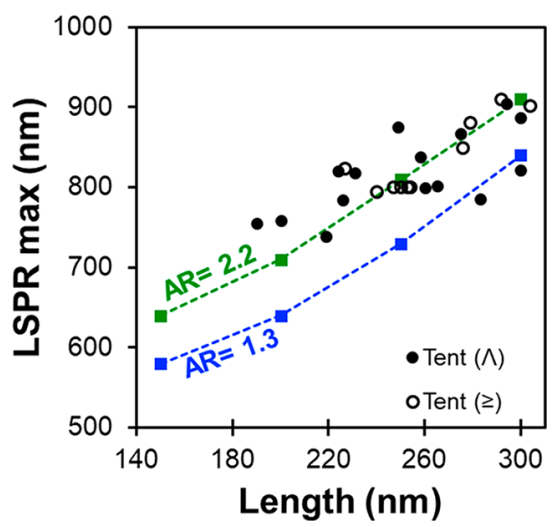

Figure 8. Comparison of numerical and experimental results on optical far-field scattering properties of tent Mg NPs. Effect of NP length on the longitudinal plasmon energy: tents in $\Lambda$ configuration are shown in filled circles, those in $\geq$, empty circles. The range of experimental AR is 1.23-2.07; fit lines to calculated longitudinal dipole energies for NPs with AR 1.3 to 2.2 are shown in blue and green, respectively.

energy peak predominantly excited with the incoming polarization along the long axis of the NP; this can be assigned to a longitudinal dipole, as it is of lowest energy. Another salient feature of the spectra is the mode around 400$600 \mathrm{~nm}$ that is excited with a polarization transverse to the long axis; this mode can be described as a transverse dipole, similar to those of $\mathrm{Au}$ and $\mathrm{Ag}$ rods. ${ }^{58,59}$ Both modes red-shift, as expected, when increasing the size of the NP. In Figure 5a, this can be seen as a shift of the longitudinal dipole from 547 $\mathrm{nm}$ to $788 \mathrm{~nm}$ and the transverse dipole from $405 \mathrm{~nm}$ to 547 $\mathrm{nm}$ as the length $(L)$ of a tent with $\mathrm{AR}=1.6$ is increased from $180 \mathrm{~nm}$ to $300 \mathrm{~nm}$. Increasing the aspect ratio of the NPs for a fixed length, $220 \mathrm{~nm}$ in Figure 5b, results in a slight red-shift of the longitudinal mode and a pronounced blue-shift of the transverse mode; that is, they become farther apart in energy as the NP becomes increasingly anisotropic, as expected. Given the direction of oscillation of the conduction electrons, and analogously to hexagonal nanoplates, ${ }^{35}$ the thickness of a folded hexagonal NP should have very little influence on the LSPR energy. DDA calculations for tent NPs (Figure S11) confirm this and show that the resonances do not move appreciably: a mere $6 \mathrm{~nm}$ shift is observed when the thickness varies from 34 to $54 \mathrm{~nm}$.

The presence of an oxide red-shifts the plasmon resonances by providing a high local refractive index environment. Because studying an oxide-free particle experimentally remains a challenge, we have devised a computational approach to include a thin, conformal oxide layer on any Wulff-generated shape, an extension of our previously published code. ${ }^{19,20}$ This enables us to properly match experimental conditions (Figure 6 onward) as well as access the magnitude of shifts expected due to this oxide. Figure S12 shows an example for a tent NP with and without a $4.5 \mathrm{~nm}$ thick oxide layer. The longitudinal mode red-shifts by $42 \mathrm{~nm}$ with the oxide, as expected from the higher dielectric environment, but the shape of the optical response remains unchanged.

The orientation of the twin plane present in the NP determines its fold angle and shape and has some effect on the far-field scattering properties, as shown for various $\mathrm{MgO}$ coated shapes in Figure 6. The tent, chair, and taco all have the same volume $\left(1.0 \times 10^{6} \mathrm{~nm}^{3}\right)$ and length $(235 \mathrm{~nm})$; their low- 


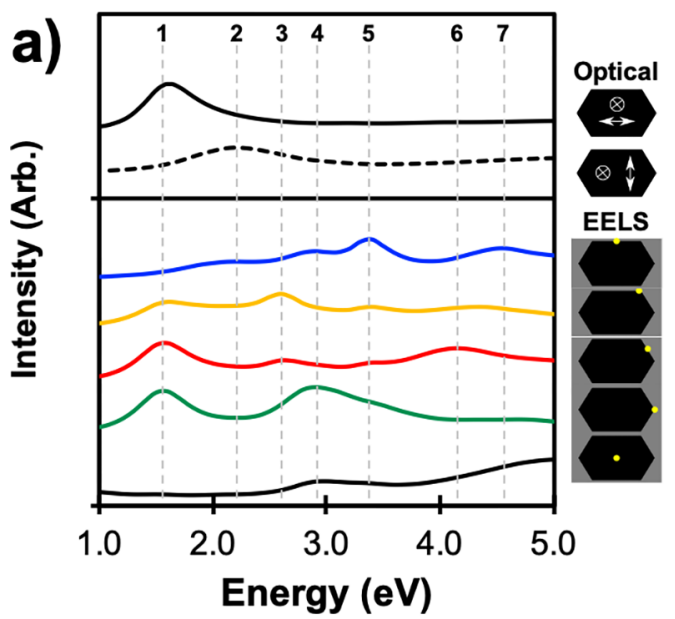

b) L= $258 \mathrm{~nm}, \mathrm{~W}=152 \mathrm{~nm}(A R$ 1.7)
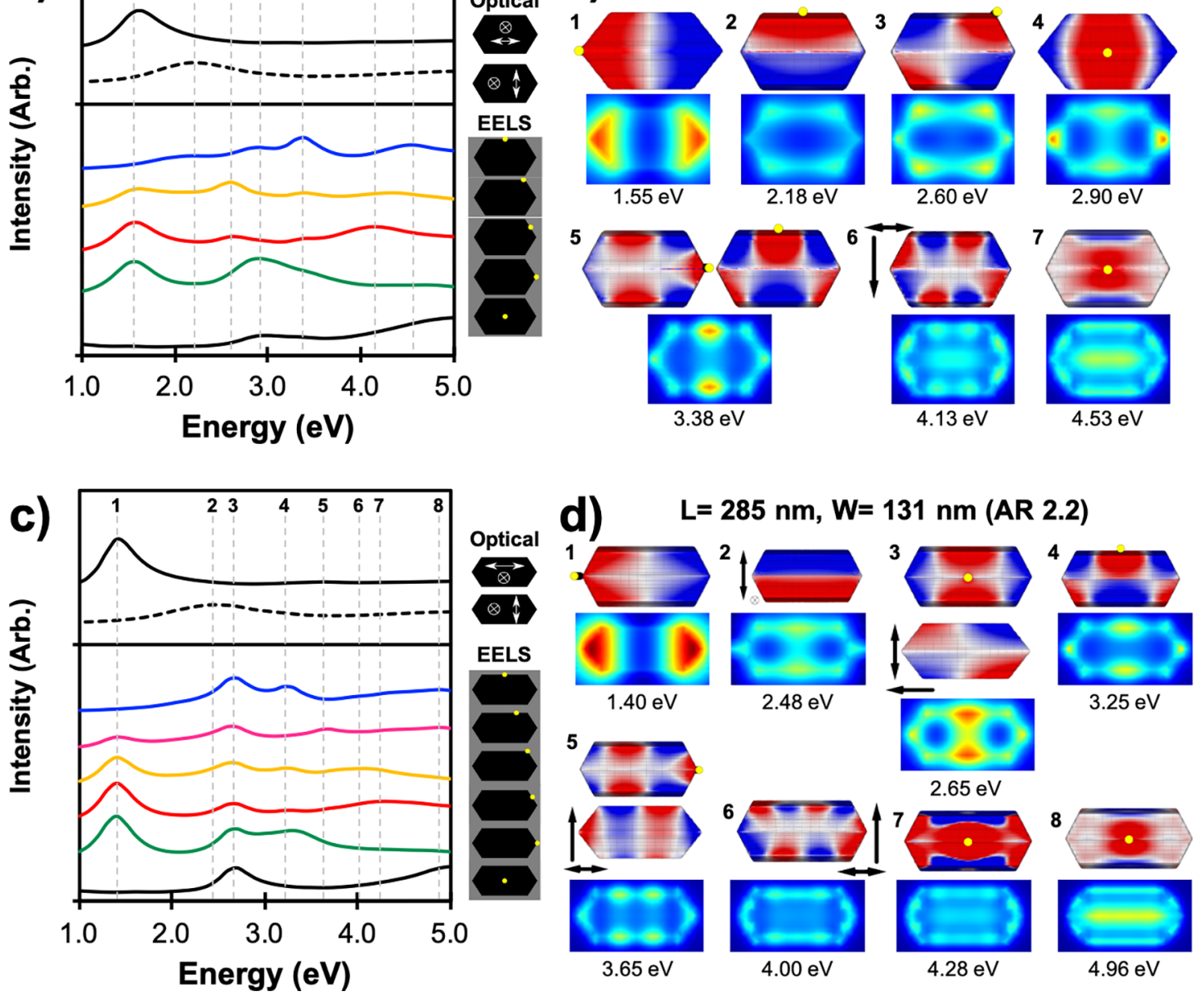

Figure 9. Numerical results from DDA and eDDA on the LSP modes of short $(A R=1.7, a, b)$ and long $(A R=2.2, c, d)$ tent Mg NPs in the $\Lambda$ configuration on a $\mathrm{Si}_{3} \mathrm{~N}_{4}$ substrate. Calculated extinction spectra from an optical excitation with polarization along the longitudinal and transverse directions are shown in solid and dashed black lines, respectively, in (a) and (c). Calculated EEL spectra on locations specified by the yellow dots are shown as solid color lines. Maps of the magnitude and sign of the resonant electric field along the surface normal $\left(E_{\perp}\right)$ at the energy indicated are shown in (b) and (d) in blue and red; these were obtained either with an electron beam excitation at the yellow dot position or optical excitation with incident direction and polarization indicated by single and double arrows, respectively. Maps of the EEL excitation probability are shown below the polarization maps in $(b)$ and $(d)$ together with the energy of the resonance; these are on the same scale within each NP.

energy LSPR is comparable. On the other hand, it is not possible to make a kite of both the same volume and length (it has a strictly fixed aspect ratio); a kite with the same volume has a longer side length $(326 \mathrm{~nm})$ than the other structures and a red-shifted LSPR (Figure 6a). A kite with the same length has a smaller volume $\left(4.1 \times 10^{5} \mathrm{~nm}^{3}\right)$ and a blue-shifted LSPR compared to the longer kite (Figure S13). Lastly, a hexagon of the same volume is shown in Figure 6e; it has a length of $196 \mathrm{~nm}$, tip-to-tip, and a blue-shifted LSPR with respect to the longer NPs; when set to the same length, its lowest energy resonance is comparable to that of the folded structures (Figure S13). The projected width of the hexagon, tent, chair, and taco decreases in this order if their lengths are constant; however this seems to have little effect on the position of the transverse excitation peak, which remains relatively constant, here just above $400 \mathrm{~nm}$. This is in agreement with previous results on bent rods for instance, where the plasmon resonances were unaffected by the bending angle. . $^{61}$
Lastly, the presence of a substrate and the NP's orientation with respect to the substrate also potentially have an effect on its plasmonic response. Similarly to the $\mathrm{MgO}$-coated hexagons previously reported, ${ }^{35}$ and unlike pristine $\mathrm{Ag}$ cubes, ${ }^{10,16}$ numerical results using optical excitation do not indicate mode splitting or significant position changes for the lowenergy modes present in the optical spectrum (Figures S12 and S14).

Experimental Results: Far-Field Optical Scattering. Single-particle scattering spectra were obtained in a dark-field optical microscope and correlated with SEM images of the same NPs. These provide experimental evidence of the plasmonic response of various NP shapes as well as a handle on size effects. Representative spectra for a kite, taco, tent, and chair (Figure 7) reveal the significant variability of the LSPR positions with respect to shape and size and its broadband nature. For all, the lowest energy mode can be attributed to the longitudinal dipole, but there is significant variability in its position, which for large NPs may exceed the operational window of our detector (low efficiency above $900 \mathrm{~nm}$ ). 


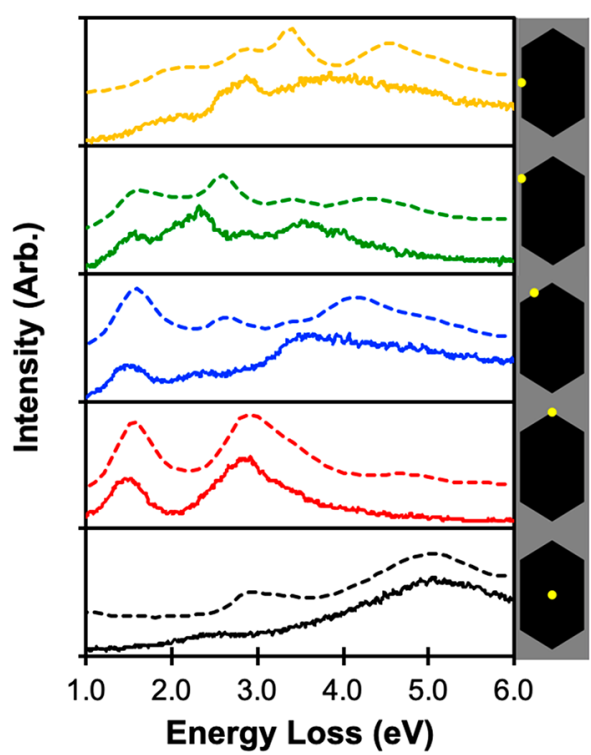

Figure 10. Local excitation of plasmon modes in a Mg NP by an electron beam. Numerical results (dashed) and experimental results (solid) at various positions for a $60 \mathrm{kV}$ electron beam impinging upon a $258 \times 152 \mathrm{~nm}(\mathrm{AR}=1.7)$ tent shape $\mathrm{NP}$ in the $\Lambda$ configuration.
Given the abundance of tent shapes, a statistically significant number of correlated measurements $(N=25)$ were assembled and yield a clear trend linking the longitudinal LSPR energy and the NP length (Figures 8 and S15). As the length of the NP increases, the energy of the resonance decreases (wavelength increases). However, there remains some noise in the data, which can in part be attributed to the influence of the AR on the longitudinal dipolar resonance (Figures 5 and S10). Numerical results for the effect of length on two extreme cases of $\mathrm{AR}, 1.3$ and 2.2, embedded in an effective refractive index chosen to mimic the substrate effect (Figure S16) match reasonably well the trend observed experimentally (Figure 8). This trend, surprisingly, appears curved; this is an artifact due to keeping a constant $\mathrm{MgO}$ thickness while changing the size of the NP, effectively slightly changing the $\mathrm{Mg}$ core shape. Remaining discrepancies can be attributed to experimental variations in the $\mathrm{Mg}$ and $\mathrm{MgO}$ thicknesses. ${ }^{20,35}$

Numerical Results: EELS and Near-Field Mode Classification. To elucidate the nature of the plasmonic modes in folded Mg NPs, detailed electronic DDA (eDDA) calculations $^{35,62}$ were performed on short (AR 1.7) and long (AR 2.2) MgO-coated tents from our Wulff code, sitting on a $\mathrm{Si}_{3} \mathrm{~N}_{4}$ substrate in the $\Lambda$ configuration. As shown in Figure 9, the calculations reveal a strong, energy-dependent subwavelength light localization stemming from their highly plasmonic nature. More precisely, the EEL spectra of both tents show many peaks that vary significantly with the position of the
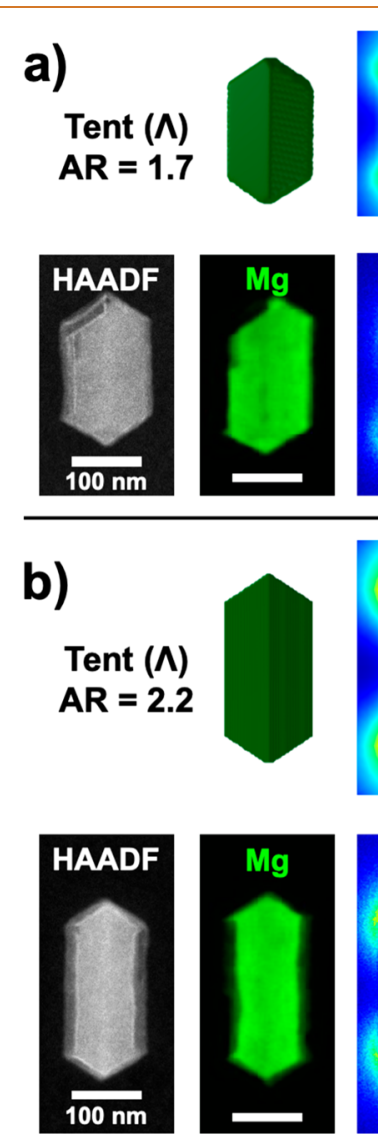

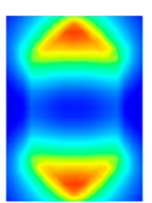

$1.55 \mathrm{eV}$
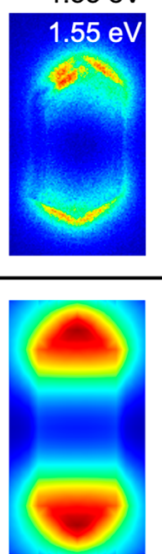

$1.40 \mathrm{eV}$
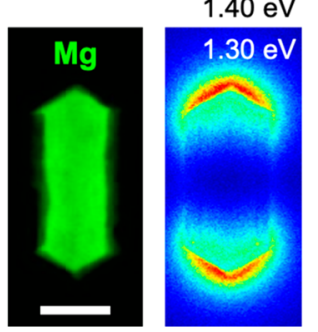

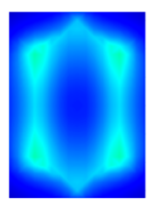

$2.18 \mathrm{eV}$
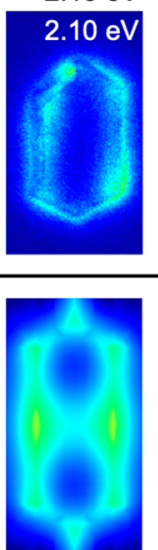

$2.48 \mathrm{eV}$

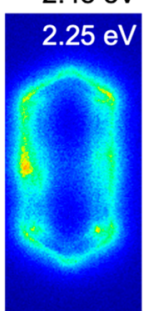

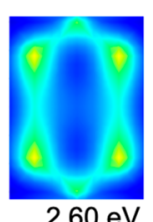
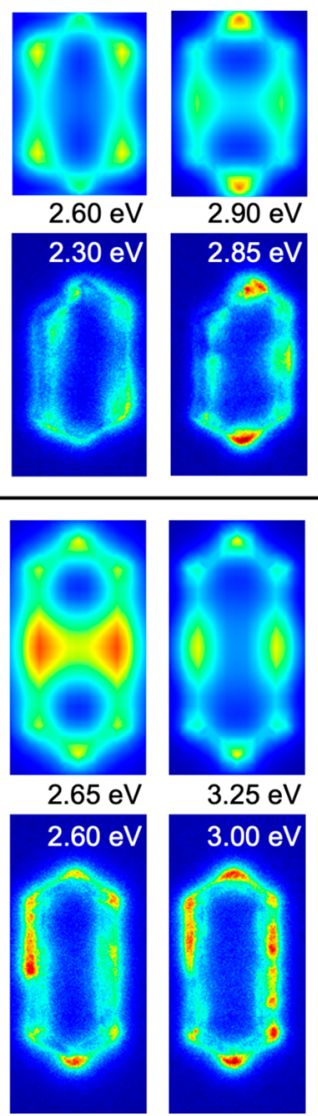
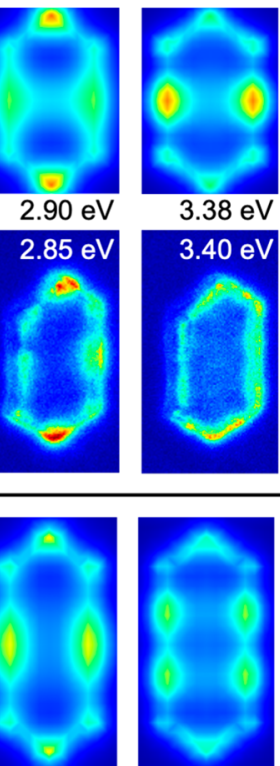

$3.38 \mathrm{eV}$
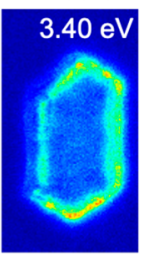

$3.25 \mathrm{eV}$
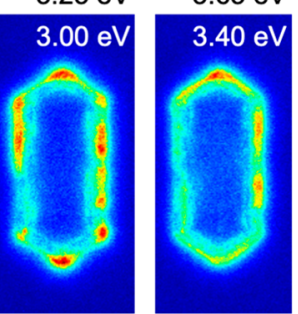
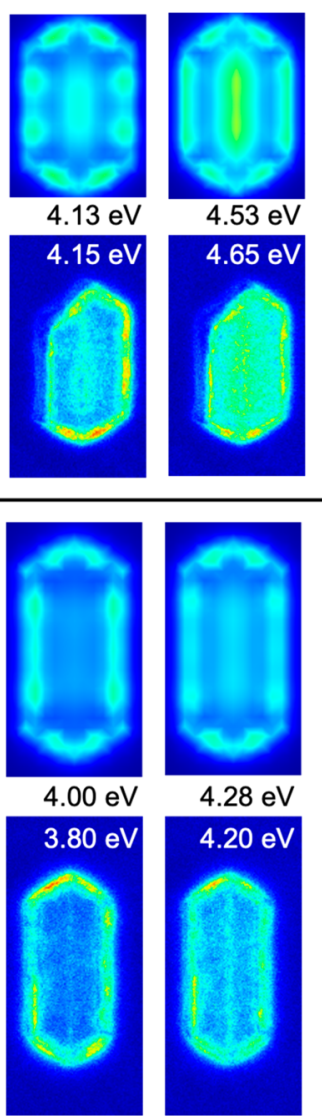
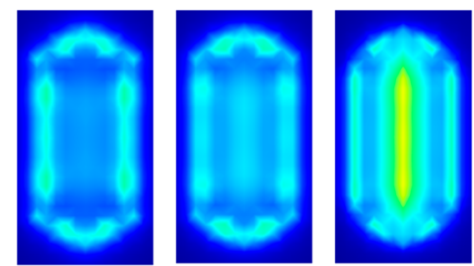

$4.96 \mathrm{eV}$

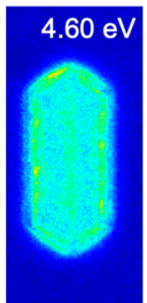

Figure 11. Calculated and experimental excitation maps of tent $\mathrm{Mg}$ NPs of AR 1.7 (a) and $2.2(\mathrm{~b})$ in the $\Lambda$ configuration with respect to the substrate. The short NP is $258 \times 152 \mathrm{~nm}$ in both the experiment and calculations, while the long NP is $285 \times 131 \mathrm{~nm}$, again in both. For each, the top row shows a schematic of the numerical shape in dark green and the calculated excitation maps also shown in Figure 9. STEMHAADF, bulk $\mathrm{Mg}$ plasmon map $(10.6 \mathrm{eV})$, and experimental maps integrated across $0.05 \mathrm{eV}$ centered at the labeled energy are shown in the bottom rows. The EELS intensity scale is the same for each mode of a given NP to allow direct comparison. 
incoming electron beam (Figure 9a,c), indicating a strong local excitation structure. Mapping the excitation probability at a specific energy informs of the spatial distribution of excitation; we couple this, in Figure $9 \mathrm{~b}$ and $\mathrm{d}$, to maps of the magnitude and sign of the resonant electric field along the surface normal $\left(E_{\perp}\right)$ to fully characterize the nature of the resonances.

In both NPs, the lowest energy peak (labeled 1 in Figure 9) corresponds to a longitudinal dipole, i.e., an oscillation along the long axis of the NP, which gives rise to a strong localized field at the tips of the NP. The energy of this mode lines up well with the peak maximum obtained for optical excitation with a polarization parallel to the long axis of the NP. Mode 2 is a weakly excited transverse dipole, even weaker in the long NP than the short one. Its oscillation gives rise to a node along the twin plane and an enhanced excitation probability on the long sides and the tips of the long sides. For the long NP, this mode is so weak under EELS excitation that it is buried in the spectral and spatial localization tail of the next, much stronger mode, though it is clear under optical excitation with polarization perpendicular to the long axis. The longitudinal dipole red-shifts from the short to the long NP as length $(L)$ is increased from $258 \mathrm{~nm}$ to $285 \mathrm{~nm}$ (matching the experimental sizes); the transverse mode blue-shifts in the long NP as expected from the increased splitting of modes discussed in relation to Figure 5 as well as the actually shorter width, $W=$ 152 and $131 \mathrm{~nm}$ for short and long NP, respectively. Beyond this, the order and overlap of modes varies between the short and long NP, such that they will be discussed separately.

In the short NP, mode 3 is predominantly excited with the beam at the transverse tips. The $E_{\perp}$ map indicates this is a quadrupole mode, with one node across the longitudinal (l) axis and one node along the transverse $(t)$ axis, or $(l, t)=(1$, $1)$. Mode 4 is a higher order longitudinal mode with 2 nodes, i.e., $(2,0)$, and high intensity at the tips and center of the transverse sides. Mode 5 has strong loss probability at the edge and tips of the transverse sides as well as the NP tips. It appears to be a $(2,1)$ mode from the $E_{\perp}$ map generated with the beam on the transverse side; however, exciting with the beam at the tips reveals a $(3,0)$ mode; both have very similar energy. A clear $(3,1)$ mode appears next (mode 6) in the EELS images; we have shown this mode in Figure $9 \mathrm{~b}$ based on optical excitation as the distortions related to beam position in the $E_{\perp}$ maps become significant in higher order, high-energy modes. At this and higher energy, modes increasingly overlap due to the smaller energy difference and large number of $(l, t)$ modes possible; at high energy, nodes also appear along the third NP axis, i.e., thickness modes, creating a plethora of $(l, t, h)$ resonances with significant spatial and spectral overlap. However, even within this complexity, one dominant mode (mode 7$)$ is clearly visible: the $(0,2)$ higher order transverse mode, strongly excited when the beam passes through the center or sides of the NP.

The modes in the long NP follow a trend similar to that of the short NP, except for the order of the modes. The mode 3 in this AR 2.2 NP is also the quadrupole $(1,1)$, which here overlaps with the $(2,0)$ as $(2,0)$ has been lowered in energy with respect to the short NP. This yields an EELS image with intensity at all of the tips. The $(3,0)$ longitudinal mode appears next (mode 5); for this we show the high symmetry obtained for numerical simulations using plane wave excitation and the distorted, but still convincing, $E_{\perp}$ map obtained from local excitation with an electron beam positioned at the tip of the NP. Beyond mode 5 there are, again, many high-energy overlapping modes, including thickness modes. Within this complexity, we present EELS images at three characteristic energies associated with weak peaks in both the numerics and experiments. Mode 6 shows edge intensity, with bright spots along the long edges and little at the tips. When excited, optically or via an electron beam, the two segments behave almost independently, supporting a range of near-degenerate modes, as captured in a characteristic E-field picture showing $(3,1)$ excitation in one segment and $(5,1)$ in the other. Mode 7 shows more high-order spots along the edges, but also a transverse component with mild intensity at the center of the NP. Mode 8 shows center and long-edge intensity and is associated with a strong transverse $(0,2)$ mode, with intensity in the third dimension on the long-edge side faces. The relative energies of the modes between the long and short tent NP are fully explained by the geometry, with increasing length lowering the energy of longitudinal modes and increasing width lowering the energy of transverse modes. This general trend accords with the pattern of standing wave frequencies $(\nu)$ for the modes in an $L \times W$ rectangle with nodes $(l, t)$, where $c$ is the speed of light:

$$
\nu^{2} \sim c^{2}\left(\frac{t^{2}}{W^{2}}+\frac{l^{2}}{L^{2}}\right)
$$

This is evident in the dipoles, where the longitudinal resonance is lower in energy in the long NP, while the transverse is lower in energy in the short NP. The energy of the $(2,0)$ with respect to the $(1,1)$, and that of the $(3,0)$ with respect to the $(2,1)$ in the long and short tents are also examples of this effect.

Experimental Results: EELS. Experimental mapping of the plasmon excitation probability with an electron beam in the ultraviolet-visible-near-infrared range was performed using a Nion UltraSTEM100MC "Hermes" monochromated STEM. Experimental spectra at different beam positions, here accumulated over a $3 \times 3$ pixels $(7.3 \times 7.3 \mathrm{~nm})$ area, are shown in Figure 10 alongside calculated spectra for a $258 \times 152 \mathrm{~nm}$ $\mathrm{Mg}$ tent NP coated with a $4.5 \mathrm{~nm}$ oxide layer, in the $\Lambda$ configuration; full point spectra also showing the $\mathrm{Mg}$ bulk plasmon can be found in Figure S17. The spectral shapes and peak positions correspond overall very well, except for an occasional small red-shift from experiment to theory that could be attributed to a number of structural or compositional factors, including the oxide layer thickness, the thickness of the $\mathrm{NP}$, and the etched irregularities in the experimental shape. Analogous, excellent matches between experimental and numerical point spectra are reported in Figures S18 and 19 for a long tent.

The excitation maps obtained for a short $(A R=1.7)$ and a long $(\mathrm{AR}=2.2)$ tent NP (Figure 11$)$, as well as various other AR (Figure S20) show a succession of energy-dependent spatial excitation patterns. To fully harness the power of our numerical calculations, the experimental data are displayed as an energy slice through the STEM-EELS data set, integrated over a narrow energy range of $0.05 \mathrm{eV}$. Gaussian fitting of modes and decomposition methods such as non-negative matrix factorization provide comparable results (shown in Figure S21 for a short tent NP; others available upon request), only with added complexity and a representational mismatch with numerics. The numerical loss probability maps shown in Figure 11 are the same as in Figure 10; the experimental map energies were guided by the position of peaks in the 
experimental spectra. The match between the calculated and experimental spatial distribution of excitation is excellent for both NPs, for both low and higher energy modes. Some variations are observed owing to the etched top left corner of the short NP, particularly visible in the mode at 3.40 and 4.15 $\mathrm{eV}$. In the long tent NP, the intensity along the long edges is systematically lower in the experiments, again likely due to etching features, this time visible on both sides of the NP; it is in fact a slightly narrower, higher AR NP (or rather the $\mathrm{Mg}$ metal is narrower) than measured in an HAADF image.

While DDA and optical scattering results did not show much effect of the substrate configuration for low-energy modes (Figure S14), eDDA and EELS provide additional tools to investigate near-field distributions that can reveal substrate effects. Figure S22 shows eDDA results including spectra at various points along a short and a long tent NP in the $\geq$ configuration and excitation maps analogous to those of Figure 9. The most striking difference from the $\Lambda$ configuration is that the spatial EELS maps are asymmetric: this is simply the geometric foreshortening of the segment rising from the substrate. Taking this into account, the low-energy modes, up to $(l, t)=(2,0)$, appear identical to those in the $\Lambda$ configuration, although the NP's more intimate contact with the substrate does lead to some additional red-shift and slight reorganization of the mode order. In particular, in the long NP, the transverse dipole becomes almost degenerate with the quadrupole, while in the short NP, the $(2,0)$ mode becomes degenerate with the quadrupole. At higher energies, splitting of the behavior of the two segments is noticeable, fitting with our previous observation that the segments can decouple at high energy in the $\Lambda$ configuration (Figure $9 \mathrm{~d}$, mode 6 ). This is particularly striking with the $(2,1)$ excitation in the short NP, which produces a strong intensity in the middle of the long edge, but at different energies for the two segments. At very high energies the exposed side facet of the proud segment becomes very bright, in accordance with our previous observations of fully three-dimensional excitations (Figure 9d, mode 8)

The elongated rods present in the sample also display plasmonic behavior. Figure 12 shows EELS maps of the excitation of three rods of various sizes ordered with increasing $\mathrm{AR}$, where $a, b$, and $c$ have $\mathrm{AR}$ of $3.4,4.5$, and 8.0, respectively. In the lowest AR rod in Figure $12 \mathrm{a}(L=260 \mathrm{~nm}, W=74 \mathrm{~nm}$, $\mathrm{AR}=3.4)$, the longitudinal $(1,0$, dipole $),(2,0)$, and $(3,0)$ modes are clearly visible. The intermediate AR rod in Figure $12 \mathrm{~b}(L=454 \mathrm{~nm}, W=87 \mathrm{~nm}, \mathrm{AR}=4.5)$ has resonances clearly attributable to longitudinal modes all significantly redshifted with respect to the AR 3.4 rod owing to the AR and length increase. Lastly, the rod with the largest $\mathrm{AR}(L=362$ $\mathrm{nm}, W=40 \mathrm{~nm}, \mathrm{AR}=8.0$ ) has clear LSPRs that are red-shifted with respect to the other NPs. The transverse dipole was not observed for any of these rods, consistent with its diminishing amplitude and increasing energy for higher AR structures. This behavior is as expected for rod-shaped, plasmonic NPs and as previously reported for various other metals. ${ }^{13-15,59,60,62,63}$ Worth noting, the classification of rods, folded shapes, and plates (Figure 2) can actually be mapped to the behavior of the transverse dipole: it is degenerate with the longitudinal dipole in plates, present but of higher energy than the longitudinal dipole in folded structures, and vanishing in intensity and of high energy in high aspect ratio rods.
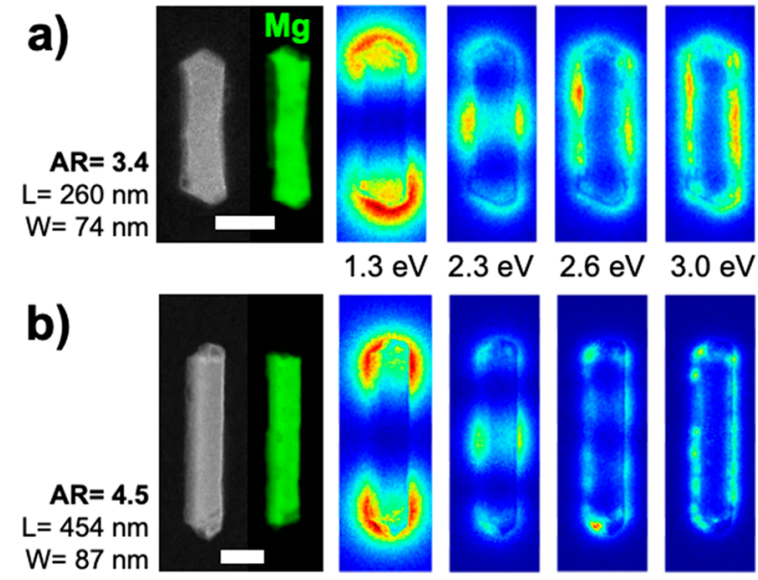

$1.3 \mathrm{eV}$

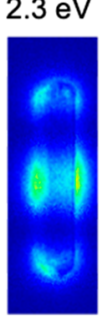

$2.6 \mathrm{eV}$

$3.0 \mathrm{eV}$
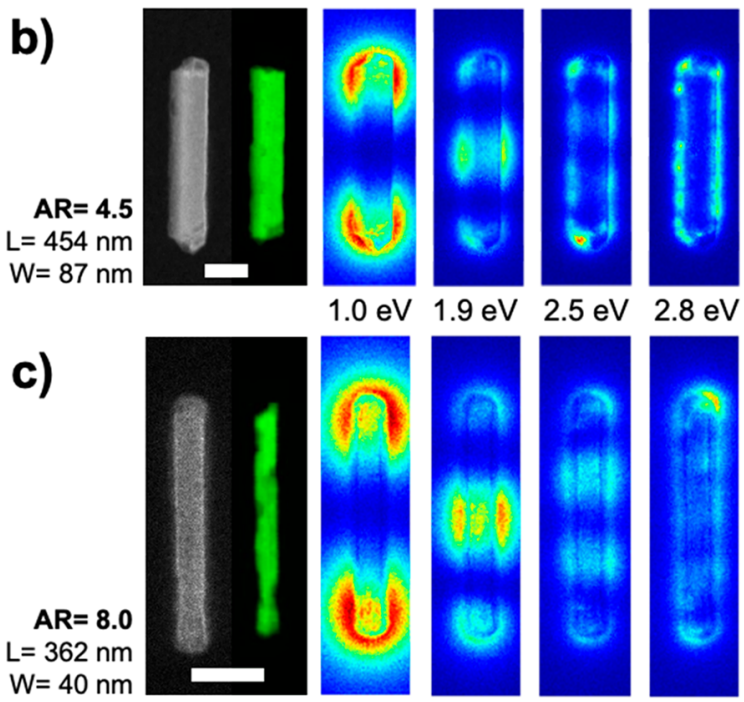

$1.0 \mathrm{eV} 1.9 \mathrm{eV}$

$2.5 \mathrm{eV} 2.8 \mathrm{eV}$
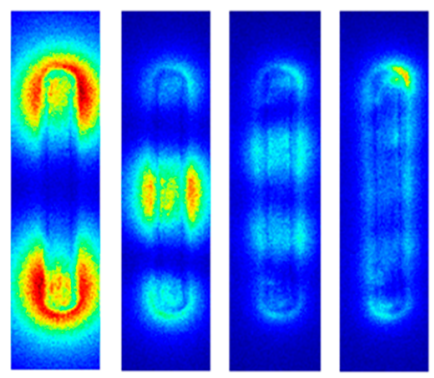

$0.9 \mathrm{eV} \quad 1.7 \mathrm{eV} \quad 2.1 \mathrm{eV} \quad 2.5 \mathrm{eV}$

Figure 12. STEM-HAADF (left), bulk plasmon maps (in green), and low-loss STEM-EELS images at the energies indicated in the figure for $\mathrm{Mg}$ nanorods of $\mathrm{AR}$, length, and width of (a) 3.4, 260 $\mathrm{nm}, 74 \mathrm{~nm},(\mathrm{~b}) 4.5,454 \mathrm{~nm}, 87 \mathrm{~nm}$, and (c) $8.0,362 \mathrm{~nm}, 40 \mathrm{~nm}$. The EELS intensity scale is the same for each mode of a given NP to allow direct comparison. Scale bars, $100 \mathrm{~nm}$.

\section{CONCLUSIONS}

Twinned shapes of $\mathrm{Mg}$, an earth-abundant plasmonic metal, were described numerically using the Wulff construction and matched to experimental results both in shape and expected abundance. These shapes, named tents, chairs, tacos, and kites, are structurally related, forming folded hexagons where the fold angle depends on the orientation of the crystallographic twin present. Rods, formed from elongated, filled structures, and single crystal plates are also present in the reaction mixture.

The plasmon resonances of the twinned shapes were investigated numerically in the far-field using DDA, showing the effect of oxide layer, size, aspect ratio, and overall shape. These results were confirmed by experimental results from single-particle far-field scattering correlated with electron microscopy. These show interpretable behavior of the longitudinal and transverse dipole.

Higher order modes and near-field distribution were numerically and experimentally determined using monochromatic electron beam excitation. Several resonant modes, from dipoles to hybrid longitudinal and transverse resonances, were fully described, and an excellent correspondence between the numerics and experiments was achieved for both short and long tent NPs, with data on intermediate length supporting these assignments. The dependence on aspect ratio and substrate orientation was explored, as was the plasmonic response of long rods which behave as expected, i.e., displaying several high-order longitudinal resonances.

These twinned shapes of earth-abundant plasmonic $\mathrm{Mg}$ provide opportunities for cheap, efficient, and biocompatible 
nanostructures for light-matter interactions. Shape and size tuning of $\mathrm{Mg}$ NPs provides a powerful way to manipulate the local electric fields and resonance energies across the nearinfrared to ultraviolet; this enables operation across a wide spectrum for potential uses in medical applications, broadband (sun)light absorption, and enhanced spectroscopies.

\section{METHODS/EXPERIMENTAL}

Chemical and Reagents. Anhydrous tetrahydrofuran (THF), anhydrous isopropanol (IPA), ethanol, naphthalene, lithium pellets, $1.0 \mathrm{M}$ di- $n$-butylmagnesium in heptane, tetraethyl orthosilicate (TEOS), and triethylamine were purchased from Sigma-Aldrich and used as supplied. Before use, all glassware was washed with aqua regia $\left(1: 3 \mathrm{HNO}_{3} / \mathrm{HCl}\right)$ and flame-dried under vacuum. (Caution: Aqua regia solutions are dangerous and should be used with extreme care; these solutions should never be stored in closed containers.)

Synthesis of $\mathbf{M g}$ Nanoparticles. Magnesium nanoparticles $(\mathrm{Mg}$ NPs) were produced by the reduction of di- $n$-butylmagnesium by lithium naphthalenide, as previously reported. ${ }^{35,39}$ A $2.12 \mathrm{~g}$ amount of naphthalene, $0.112 \mathrm{~g}$ of lithium, and $20 \mathrm{~mL}$ of anhydrous THF were added to a Schlenk flask under an argon atmosphere and sonicated for $1 \mathrm{~h}$, forming a deep green solution of lithium naphthalenide. A $23 \mathrm{~mL}$ amount of anhydrous THF and $7 \mathrm{~mL}$ of di- $n$-butylmagnesium in heptane $(1.0 \mathrm{M})$ were then added under an argon atmosphere and left to stir for $16 \mathrm{~h}$ with a magnetic stirbar.

The reaction was quenched by addition of $20 \mathrm{~mL}$ of anhydrous IPA, and the product recovered by centrifugation and redispersion in anhydrous THF twice and anhydrous IPA twice to remove residual lithium, naphthalene, and organic byproducts. Organic byproducts on the particles were further cleaned by adding $0.6 \mathrm{~mL}$ of TEOS in ethanol solution $(9 \mathrm{mM})$ and $0.2 \mathrm{~mL}$ of triethylamine to a suspension of Mg NPs ( $1 \mathrm{~mL}$ ) diluted with $5 \mathrm{~mL}$ of ethanol, and left to react for 16-20 h. The NPs were recovered by centrifugation and redispersed in $2 \mathrm{~mL}$ of anhydrous ethanol. Silica shells were not observed in imaging or elemental mapping.

Characterization. Samples were drop cast on $\mathrm{Si}$ wafers for SEM imaging performed on a Quanta-650F field emission gun scanning electron microscope operated at $5 \mathrm{kV}$ and equipped with an ETD detector for SE imaging. TEM and STEM analyses were performed on NPs drop cast on a Cu-supported lacey ultrathin carbon membrane. TEM, STEM, STEM-EELS for composition mapping, and STEMEDS were acquired at $200 \mathrm{kV}$ on a FEI Osiris STEM equipped with a Bruker Super-X quadruple EDS detector and a Gatan Enfinium ER 977 electron spectrometer. STEM-EDS maps were obtained by integrating the $\mathrm{K} \alpha$ lines of $\mathrm{Mg}(1.25 \mathrm{eV})$ and $\mathrm{O}(0.53 \mathrm{eV})$. Tilt series for tomography were acquired with a $1^{\circ}$ step from $-70^{\circ}$ to $-60^{\circ}$, and $60^{\circ}$ to $70^{\circ}$, and a $2^{\circ}$ step from $-60^{\circ}$ to $60^{\circ}$, and were aligned using phase correlation, then reconstructed using the Simultaneous Iterative Reconstruction Technique (SIRT) implemented by TomoPy. ${ }^{64}$ A 3D voxel intensity threshold was applied to determine the region of interest, and subsequently an isosurface was fitted to obtain the final extracted NP shape.

Low-loss EEL spectra were obtained, for NPs deposited on $10 \mathrm{~nm}$ thick $\mathrm{Si}_{3} \mathrm{~N}_{4}$ membrane windows baked in vacuum at $75^{\circ} \mathrm{C}$, on a Nion UltraSTEM 100MC "Hermes" microscope, a dedicated STEM equipped with a cold field electron emitter, and Nion's ultrahigh resolution ground-potential monochromator that routinely delivers energy resolutions better than $15 \mathrm{meV}$ (as determined from the full width at half-maximum of the zero loss peak, ZLP fwhm). The microscope was operated at $60 \mathrm{kV}$, and the probe forming optics were configured to provide an electron probe of $31 \mathrm{mrad}$ convergence semiangle and a probe current of $50 \mathrm{pA}$ before closing the monochromator slit, corresponding to a probe size of approximately 1 Å.

Low-loss EEL spectra were recorded on a Gatan Enfinium ERS spectrometer, with custom lens power supplies optimized for highenergy resolution. A 44 mrad semiangle EELS entrance aperture was used for all data. The system's monochromator slit was closed as required by the desired energy resolution, resulting here in a $30 \mathrm{meV}$
ZLP fwhm and 5-7 pA current. The Nion's HAADF imaging detector was set at an angular range of 90-195 mrad.

Single-particle optical dark-field measurements were carried out on Mg NPs drop cast on a glass coverslip in air. The scattering spectra were obtained on an optical microscope described elsewhere. ${ }^{65}$ In brief, it is equipped with a halogen lamp, dark-field condenser (numerical aperture, NA, 0.85-0.95), 100× oil immersion objective (Variable NA set to $<0.8$ ), Princeton Instruments Isoplane spectrometer (50 grooves $/ \mathrm{mm}$ grating), and ProEM $1024 \times 1024$ pixels electron multiplied charge coupled device (EMCCD). The exposure time was set to $1 \mathrm{~s}$ with 4 frames accumulated per position.

Numerical Methods. Optical scattering spectra were obtained numerically in the discrete dipole approximation using DDSCAT. ${ }^{57,66}$ The frequency-dependent refractive index of metallic $\mathrm{Mg}$ was taken from Palik, ${ }^{67}$ and the ambient, $\mathrm{MgO}$, and glass refractive indexes were set to 1.0,1.7, and 1.5, respectively. All calculations were carried out with dipole distances from 1.0 to $2.2 \mathrm{~nm}$, as the number of dipoles varies from 200 000 to 600000 . Details can be found in Table S1.

EELS calculations were performed using eDDA, ${ }^{62}$ a version of $\operatorname{DDSCAT}^{57,68}$ modified to replace the plane wave stimulation with a stimulation with a swift electron beam. In all cases, NPs consisted of a $\mathrm{Mg}$ core with a $4.5 \mathrm{~nm} \mathrm{MgO}$ shell, generated via our Wulff code. In order to include the effect of the experimental TEM support film, the NPs sat (in $\Lambda$ and $\geq$ configuration) on a $10 \mathrm{~nm}$ thick $\mathrm{Si}_{3} \mathrm{~N}_{4}$ substrate (refractive index 2.05) that extended at least $40 \mathrm{~nm}$ beyond the edges of the NP. The calculations used a $2 \mathrm{~nm}$ dipole lattice, requiring around 300000 dipoles for each shape. The DDA calculations shown within the EELS figures also use the $\mathrm{Si}_{3} \mathrm{~N}_{4}$ substrate, to aid comparison with their eDDA counterparts.

\section{ASSOCIATED CONTENT}

\section{Supporting Information}

The Supporting Information is available free of charge at https://pubs.acs.org/doi/10.1021/acsnano.0c01427.

High- and low-magnification SEM and TEM images, additional numerical results, raw data and characterization of STEM-EDS and STEM-EELS line profiles and maps, non-negative matrix factorization analyses comparison with Gaussian fitting of STEM-EELS data; shapes, dimensions, DDA parameters, and Wulff parameters for all theoretical NPs presented here (PDF)

\section{AUTHOR INFORMATION}

\section{Corresponding Author}

Emilie Ringe - Department of Materials Science and Metallurgy and Department of Earth Sciences, University of Cambridge, Cambridge, United Kingdom CB3 OFS; 이이.org/00000003-3743-9204; Phone: +44 (0)1223 334330; Email: er407@cam.ac.uk; Fax: +44 (0)1223 334567

\section{Authors}

Jérémie Asselin - Department of Materials Science and Metallurgy and Department of Earth Sciences, University of Cambridge, Cambridge, United Kingdom CB3 OFS; (1) orcid.org/0000-0002-6220-6739

Christina Boukouvala - Department of Materials Science and Metallurgy and Department of Earth Sciences, University of Cambridge, Cambridge, United Kingdom CB3 OFS

Elizabeth R. Hopper - Department of Materials Science and Metallurgy, Department of Earth Sciences, and Department of Chemical Engineering and Biotechnology, University of Cambridge, Cambridge, United Kingdom CB3 OFS

Quentin M. Ramasse - School of Chemical and Process Engineering and School of Physics and Astronomy, University of 
Leeds, Leeds, United Kingdom LS2 9JT; SuperSTEM, SciTech Daresbury Science and Innovation Campus, Warrington, United Kingdom WA4 4AD; 이이이.org/0000-0001-7466-2283

John S. Biggins - Department of Engineering, University of Cambridge, Cambridge, United Kingdom CB2 1PZ; (1) orcid.org/0000-0002-7452-2421

Complete contact information is available at: https://pubs.acs.org/10.1021/acsnano.0c01427

\section{Author Contributions}

$\triangle_{\mathrm{J}}$. Asselin and C. Boukouvala contributed equally.

\section{Notes}

The authors declare no competing financial interest.

\section{ACKNOWLEDGMENTS}

Support for this project was provided by the EU Framework Programme for Research and Innovation Horizon 2020 (ERC Starting Grant SPECs 804523). J.A. wishes to acknowledge financial support from Natural Sciences and Engineering Research Council of Canada and Fonds de Recherche Québec-Nature et Technologies postdoctoral fellowships (BP and B3X programs). C.B. is thankful for funding from the Engineering and Physical Sciences Research Council (Standard Research Studentship (DTP) EP/R513180/1), and E.R.H. for support from the EPSRC NanoDTC Cambridge (EP/L015978/1). Q.M.R. acknowledges that SuperSTEM is the UK National Research Facility for Advanced Electron Microscopy, supported by the Engineering and Physical Sciences Research Council (EPSRC). J.S.B. acknowledges the UKRI Future Leaders Fellowship program, grant number MR/S017186/1.

\section{REFERENCES}

(1) Willets, K. A.; Van Duyne, R. P. Localized Surface Plasmon Resonance Spectroscopy and Sensing. Annu. Rev. Phys. Chem. 2007, 58, 267-297.

(2) Mayer, K. M.; Hafner, J. H. Localized Surface Plasmon Resonance Sensors. Chem. Rev. 2011, 111, 3828-3857.

(3) Sharma, B.; Frontiera, R. R.; Henry, A.-I.; Ringe, E.; Van Duyne, R. P. SERS: Materials, Applications, and the Future. Mater. Today 2012, 15, 16-25.

(4) Linic, S.; Aslam, U.; Boerigter, C.; Morabito, M. Photochemical Transformations on Plasmonic Metal Nanoparticles. Nat. Mater. 2015, 14, 567-576.

(5) Brongersma, M. L.; Halas, N. J.; Nordlander, P. PlasmonInduced Hot Carrier Science and Technology. Nat. Nanotechnol. 2015, 10, 25-34.

(6) Swearer, D. F.; Zhao, H.; Zhou, L.; Zhang, C.; Robatjazi, H.; Martirez, J. M. P.; Krauter, C. M.; Yazdi, S.; McClain, M. J.; Ringe, E.; Carter, E. A.; Nordlander, P.; Halas, N. J. Heterometallic AntennaReactor Complexes for Photocatalysis. Proc. Natl. Acad. Sci. U. S. A. 2016, 113, 8916-8920.

(7) Baffou, G.; Quidant, R. Nanoplasmonics for Chemistry. Chem. Soc. Rev. 2014, 43, 3898-3907.

(8) Abadeer, N. S.; Murphy, C. J. Recent Progress in Cancer Thermal Therapy Using Gold Nanoparticles. J. Phys. Chem. C 2016, 120, 4691-4716.

(9) Kelly, K. L.; Coronado, E.; Zhao, L. L.; Schatz, G. C. The Optical Properties of Metal Nanoparticles: The Influence of Size, Shape, and Dielectric Environment. J. Phys. Chem. B 2003, 107, 668677.

(10) Ringe, E.; McMahon, J. M.; Sohn, K.; Cobley, C.; Xia, Y.; Huang, J.; Schatz, G. C.; Marks, L. D.; Van Duyne, R. P. Unraveling the Effects of Size, Composition, and Substrate on the Localized Surface Plasmon Resonance Frequencies of Gold and Silver
Nanocubes: A Systematic Single-Particle Approach. J. Phys. Chem. C 2010, 114, 12511-12516.

(11) Ringe, E.; Langille, M. R.; Sohn, K.; Zhang, J.; Huang, J.; Mirkin, C. A.; Van Duyne, R. P.; Marks, L. D. Plasmon Length: A Universal Parameter to Describe Size Effects in Gold Nanoparticles. J. Phys. Chem. Lett. 2012, 3, 1479-1483.

(12) Rossouw, D.; Couillard, M.; Vickery, J.; Kumacheva, E.; Botton, G. A. Multipolar Plasmonic Resonances in Silver Nanowire Antennas Imaged with a Subnanometer Electron Probe. Nano Lett. 2011, 11, $1499-1504$

(13) Imura, K.; Nagahara, T.; Okamoto, H. Near-Field Optical Imaging of Plasmon Modes in Gold Nanorods. J. Chem. Phys. 2005, $122,154701$.

(14) Payne, E. K.; Shuford, K. L.; Park, S.; Schatz, G. C.; Mirkin, C. A. Multipole Plasmon Resonances in Gold Nanorods. J. Phys. Chem. B 2006, 110, 2150-2154.

(15) Bryant, G. W.; García De Abajo, F. J.; Aizpurua, J. Mapping the Plasmon Resonances of Metallic Nanoantennas. Nano Lett. 2008, 8, 631-636.

(16) Nicoletti, O.; De La Peña, F.; Leary, R. K.; Holland, D. J.; Ducati, C.; Midgley, P. A. Three-Dimensional Imaging of Localized Surface Plasmon Resonances of Metal Nanoparticles. Nature 2013, 502, 80-84.

(17) Wulff, G. On the Question of Speed of Growth and Dissolution of Crystal Surfaces. Zeitschrift für Krist. 1901, 37, 449.

(18) Marks, L. D. Modified Wulff Constructions for Twinned Particles. J. Cryst. Growth 1983, 61, 556-566.

(19) Ringe, E.; Van Duyne, R. P.; Marks, L. D. Kinetic and Thermodynamic Modified Wulff Constructions for Twinned Nanoparticles. J. Phys. Chem. C 2013, 117, 15859-15870.

(20) Boukouvala, C.; Ringe, E. Wulff-Based Approach to Modeling the Plasmonic Response of Single Crystal, Twinned, and Core-Shell Nanoparticles. J. Phys. Chem. C 2019, 123, 25501-25508.

(21) Marks, L. D. Experimental Studies of Small Particle Structures. Rep. Prog. Phys. 1994, 57, 603-649.

(22) Lu, X.; Rycenga, M.; Skrabalak, S. E.; Wiley, B.; Xia, Y. Chemical Synthesis of Novel Plasmonic Nanoparticles. Annu. Rev. Phys. Chem. 2009, 60, 167-192.

(23) Zhang, J.; Li, S.; Wu, J.; Schatz, G. C.; Mirkin, C. A. PlasmonMediated Synthesis of Silver Triangular Bipyramids. Angew. Chem., Int. Ed. 2009, 48, 7787-7791.

(24) Ino, S. Epitaxial Growth of Metals on Rocksalt Faces Cleaved in Vacuum. II. Orientation and Structure of Gold Particles Formed in Ultrahigh Vacuum. J. Phys. Soc. Jpn. 1966, 21, 346-362.

(25) Sánchez-Iglesias, A.; Pastoriza-Santos, I.; Pérez-Juste, J.; Rodríguez-González, B.; García De Abajo, F. J.; Liz-Marzán, L. M. Synthesis and Optical Properties of Gold Nanodecahedra with Size Control. Adv. Mater. 2006, 18, 2529-2534.

(26) Sun, Y.; Xia, Y. Shape-Controlled Synthesis of Gold and Silver Nanoparticles. Science (Washington, DC, U. S.) 2002, 298, 21762179.

(27) Jana, N. R.; Gearheart, L.; Murphy, C. J. Seed-Mediated Growth Approach for Shape-Controlled Synthesis of Spheroidal and Rod-Like Gold Nanoparticles Using a Surfactant Template. Adv. Mater. 2001, 13, 1389-1393.

(28) Xia, Y.; Xiong, Y.; Lim, B.; Skrabalak, S. E. Shape-Controlled Synthesis of Metal Nanocrystals: Simple Chemistry Meets Complex Physics? Angew. Chem., Int. Ed. 2009, 48, 60-103.

(29) Grzelczak, M.; Pérez-Juste, J.; Mulvaney, P.; Liz-Marzán, L. M. Shape Control in Gold Nanoparticle Synthesis. Chem. Soc. Rev. 2008, 37, 1783-1791.

(30) Personick, M. L.; Langille, M. R.; Zhang, J.; Mirkin, C. A. Shape Control of Gold Nanoparticles by Silver Underpotential Deposition. Nano Lett. 2011, 11, 3394-3398.

(31) Marks, L. D. Surface Structure and Energetics of Multiply Twinned Particles. Philos. Mag. A 1984, 49, 81-93.

(32) Baletto, F.; Ferrando, R. Structural Properties of Nanoclusters: Energetic, Thermodynamic, and Kinetic Effects. Rev. Mod. Phys. 2005, 77, 371-423. 
(33) Sterl, F.; Strohfeldt, N.; Walter, R.; Griessen, R.; Tittl, A.; Giessen, H. Magnesium as Novel Material for Active Plasmonics in the Visible Wavelength Range. Nano Lett. 2015, 15, 7949-7955.

(34) Jeong, H. H.; Mark, A. G.; Fischer, P. Magnesium Plasmonics for UV Applications and Chiral Sensing. Chem. Commun. 2016, 52, 12179-12182.

(35) Biggins, J. S.; Yazdi, S.; Ringe, E. Magnesium Nanoparticle Plasmonics. Nano Lett. 2018, 18, 3752-3758.

(36) Gutierrez, Y.; Ortiz, D.; Sanz, J. M.; Saiz, J. M.; Gonzalez, F.; Everitt, H. O.; Moreno, F. How an Oxide Shell Affects the Ultraviolet Plasmonic Behavior of $\mathrm{Ga}, \mathrm{Mg}$, and $\mathrm{Al}$ Nanostructures. Opt. Express 2016, 24, 20621-20631.

(37) Duan, X.; Liu, N. Magnesium for Dynamic Nanoplasmonics. Acc. Chem. Res. 2019, 52, 1979-1989.

(38) Blaber, M. G.; Arnold, M. D.; Ford, M. J. A Review of the Optical Properties of Alloys and Intermetallics for Plasmonics. J. Phys.: Condens. Matter 2010, 22, 143201.

(39) Asselin, J.; Boukouvala, C.; Wu, Y.; Hopper, E. R.; Collins, S. M.; Biggins, J. S.; Ringe, E. Decoration of Plasmonic $\mathrm{Mg}$ Nanoparticles by Partial Galvanic Replacement Decoration of Plasmonic Mg Nanoparticles by Partial Galvanic Replacement. J. Chem. Phys. 2019, 244708, 1-8.

(40) Rieke, R. D. Preparation of Organometallic Compounds from Highly Reactive Metal Powders. Science (Washington, DC, U. S.) 1989, 246, 1260-1264.

(41) Song, M. R.; Chen, M.; Zhang, Z. J. Preparation and Characterization of Mg Nanoparticles. Mater. Charact. 2008, 59, 514-518.

(42) Jeon, K. J.; Moon, H. R.; Ruminski, A. M.; Jiang, B.; Kisielowski, C.; Bardhan, R.; Urban, J. J. Air-Stable Magnesium Nanocomposites Provide Rapid and High-Capacity Hydrogen Storage without Using Heavy-Metal Catalysts. Nat. Mater. 2011, 10, 286290.

(43) Viyannalage, L.; Lee, V.; Dennis, R. V.; Kapoor, D.; Haines, C. D.; Banerjee, S. From Grignard's Reagents to Well-Defined $\mathrm{Mg}$ Nanostructures: Distinctive Electrochemical and Solution Reduction Routes. Chem. Commun. 2012, 48, 5169-5171.

(44) Norberg, N. S.; Arthur, T. S.; Fredrick, S. J.; Prieto, A. L. SizeDependent Hydrogen Storage Properties of $\mathrm{Mg}$ Nanocrystals Prepared from Solution. J. Am. Chem. Soc. 2011, 133, 10679-10681.

(45) Aguey-Zinsou, K. F.; Ares-Fernández, J. R. Synthesis of Colloidal Magnesium: A Near Room Temperature Store for Hydrogen. Chem. Mater. 2008, 20, 376-378.

(46) Garza-Rodríguez, L. A.; Kharisov, B. I.; Kharissova, O. V. Overview on the Synthesis of Activated Micro- and Nanostructurized Rieke Metals: History and Present State. Synth. React. Inorg. M. 2009, 39, 270-290.

(47) Ohno, T.; Yamauchi, K. Magnesium Twinned Particles Grown in Inert Gases. Jpn. J. Appl. Phys. 1981, 20, 1385-1391.

(48) Liu, W.; Aguey-Zinsou, K.-F. Size Effects and Hydrogen Storage Properties of $\mathrm{Mg}$ Nanoparticles Synthesised by an Electroless Reduction Method. J. Mater. Chem. A 2014, 2, 9718-9726.

(49) Partridge, P. G. The Crystallography and Deformation Modes of Hexagonal Close-Packed Metals. Metall. Rev. 1967, 12, 169-194.

(50) Christian, J. W. Deformation Twinning (Part II). In The Theory of Transformations in Metals and Alloys; Elsevier Science: Oxford, 2002; pp 859-951.

(51) Kopač Lautar, A.; Kopač, D.; Rejec, T.; Bančič, T.; Dominko, R. Morphology Evolution of Magnesium Facets: DFT and KMC Simulations. Phys. Chem. Chem. Phys. 2019, 21, 2434-2442.

(52) Ringe, E.; Van Duyne, R. P.; Marks, L. D. Wulff Construction for Alloy Nanoparticles. Nano Lett. 2011, 11, 3399-3403.

(53) Roosen, A. R.; McCormack, R. P.; Carter, W. C. Wulffman: A Tool for the Calculation and Display of Crystal Shapes. Comput. Mater. Sci. 1998, 11, 16-26.

(54) Li, W.; Li, C.; Zhou, C.; Ma, H.; Chen, J. Metallic Magnesium Nano/Mesoscale Structures: Their Shape-Controlled Preparation and $\mathrm{Mg} /$ Air Battery Applications. Angew. Chem., Int. Ed. 2006, 45, 60096012.
(55) Pei, Z.; Zhang, X.; Hickel, T.; Friák, M.; Sandlöbes, S.; Dutta, B.; Neugebauer, J. Atomic Structures of Twin Boundaries in Hexagonal Close-Packed Metallic Crystals with Particular Focus on Mg. npj Comput. Mater. 2017, 3, 1-6.

(56) Serra, A.; Bacon, D. J. Computer Simulation of Twinning Dislocation in Magnesium Using a Many-Body Potential. Philos. Mag. A 1991, 63, 1001-1012.

(57) Draine, B. T.; Flatau, P. J. Discrete-Dipole Approximation for Scattering Calculations. J. Opt. Soc. Am. A 1994, 11, 1491-1499.

(58) Brioude, A.; Jiang, X. C.; Pileni, M. P. Optical Properties of Gold Nanorods: DDA Simulations Supported by Experiments. J. Phys. Chem. B 2005, 109, 13138-13142.

(59) Mahmoud, M. A.; El-Sayed, M. A. Different Plasmon Sensing Behavior of Silver and Gold Nanorods. J. Phys. Chem. Lett. 2013, 4, $1541-1545$.

(60) Rossouw, D.; Botton, G. A. Plasmonic Response of Bent Silver Nanowires for Nanophotonic Subwavelength Waveguiding. Phys. Rev. Lett. 2013, 110, 1-5.

(61) Boudarham, G.; Feth, N.; Myroshnychenko, V.; Linden, S.; García De Abajo, J.; Wegener, M.; Kociak, M. Spectral Imaging of Individual Split-Ring Resonators. Phys. Rev. Lett. 2010, 105, 1-4.

(62) Bigelow, N. W.; Vaschillo, A.; Iberi, V.; Camden, J. P.; Masiello, D. J. Characterization of the Electron- and Photon-Driven Plasmonic Excitations of Metal Nanorods. ACS Nano 2012, 6, 7497-7504.

(63) Clark, B. D.; Jacobson, C. R.; Lou, M.; Yang, J.; Zhou, L.; Gottheim, S.; DeSantis, C. J.; Nordlander, P.; Halas, N. J. Aluminum Nanorods. Nano Lett. 2018, 18, 1234-1240.

(64) Gürsoy, D.; De Carlo, F.; Xiao, X.; Jacobsen, C. TomoPy: A Framework for the Analysis of Synchrotron Tomographic Data. J. Synchrotron Radiat. 2014, 21, 1188-1193.

(65) Kumar, A.; Villarreal, E.; Zhang, X.; Ringe, E. Micro-Extinction Spectroscopy (MExS): A Versatile Optical Characterization Technique. Adv. Struct. Chem. Imaging 2018, 4, 8.

(66) Flatau, P. J.; Draine, B. T. Fast Near Field Calculations in the Discrete Dipole Approximation for Regular Rectilinear Grids. Opt. Express 2012, 20, 1247-1252.

(67) Palik, E. D. Introduction to the Data of Several Metals. In Handbook of Optical Constants of Solids; Academic Press: Cambridge, MA, 1998; pp 238-286.

(68) Flatau, P. J.; Draine, B. T. Light Scattering by Hexagonal Columns in the Discrete Dipole Approximation. Opt. Express 2014, 22, 21834-21846. 